\title{
Sur les anneaux tels que tout produit de copies d'un module quasi-injectif soit un module quasi-injectif (*).
}

\author{
Claude Tisseron (Lyon)
}

\begin{abstract}
Résumé. - Un anneau A tel que tout produit de copies d'un A-module quasi-injectif soit un $A$-module quasi-injectif est appelé un Q-anneau. Pour un anneau $A$ tout $A$-modnle quasiinjectif est injectif si et sexlement si $A$ est un $Q$-anneau dont le radical de Jacobson $R(A)$ est nul. On étudie les propriétés des Q-anneaux d'abord en général puis lorsqu'ils sont noethériens. Dans ce cas on s'intéresse d'une part au complété d'un Q-anneau semi-local $A$ pour la topologie $R(A)$-adique et d'autre part on caractérise les Q-anneaux locaux dont on donne des exemples.
\end{abstract}

Certains problèmes généraux consistent à caractériser les anneaux $A$ tels que la classe de $A$-modules ayant une propriété $P$ donnée soit stable par produit (resp. somme directe). Par exemple dans [6], Chase caractérise les anneaux tels que tout produit de modules plats soit un module plat et les anneaux tels que tout produit de modules projectifs soit un module projectif. Dans l'étude de ces problèmes on peut parfois se limiter aux anneaux tels que tout produit (respectivement toute somme) de copies d'un module ayant la propriété $P$ ait la propriété $P$ (cf. [6], théorèmes 2.1 et 3.3 ); un tel module est appelé $\Pi-P$ (resp. $\Sigma-P$ ). On s'est intéressé à des problèmes analogues pour des propriétés $P$ moins classiques: la quasi-injectivité et la quasi-projectivité. La $\Sigma$-quasi-injectivité est étudiée dans [12] et la $\Sigma$-quasi-projectivité dans [12] et [32]. La $\Sigma$-quasi-projectivité est étudiée dans [22], [28] et [32]. Dans la partie I de cet article, on rappelle d'abord quelques résultats sur la quasi-injectivité puis on introduit les anneaux tels que tout module quasi-injectif soit $\Pi$-quasi-injectif, dont l'étude est le but de ce travail. Ces anneaux sont appelés $Q$-anneaux dans [32]. L'étude de ces anneaux fait intervenir une notion un peu plus générale, celle de 01 anneau, définie de la façon suivante: si $E$ est l'enveloppe injective d'une somme d'un $A$-module simple de chaque type, alors $A$ est un $O 1$ anneau si tout sous-module quasi-injectif de $E$ est $\Pi$-quasi-injectif. Si $A$ est un $C 1$ anneau, il $y$ a une dualité entre certains sous-modules quasi-injectifs de $E$ et certains idéaux de $A$ (II. proposition 2.6) qui rappelle un résultat de [20] (théorème 4.2).

Les $C 1$ anneaux et les $Q$-anneaux sont étudiés parallèlement dans la partie II où on donne des propriétés de stabilité de ces anneaux ainsi que des caratérisations dans le cas où leur radical de Jacobson est nul. On met également en évidence dans cette partie certains caractères noethériens des $Q$-anneaux, et on rappelle le fait

(*) Entrata in Redazione il 1 agosto 1973. 

dû à Renault [27] que si $A$ est un $Q$-anneau de radical $R$ alors $A / \bigcap_{n \geqslant 0} \mathcal{R}^{n}$ est un anneau
noethérien.

Ceci nous amène dans la partie III, à nous limiter à l'étude des $O 1$ anneaux et des $Q$-anneaux $A$, noethériens et tels que $\bigcap_{n \geqslant 0} \mathcal{R}^{n}=0$. Dans une première partie on montre que le complété d'un $C 1$ anneau noethérien $A$ de radical $R$ tel que $A / \Re$ soit semi-simple et $\bigcap_{n \geqslant 0} \mathcal{R}^{n}=0$ se comporte à peu près comme un anneau de Zariski. Dans une seconde partie on caractérise certains $Q$-anneaux noethériens, ce qui permet entre autre de montrer qu'un anneau de Dedekind premier borné $A$ est un $Q$-anneau si et seulement si $A / \mathcal{R}$ est un anneau simple.

\section{I. - Modules quasi-injectifs, modules $\pi$-quasi-injectifs.}

\section{1. - Notations.}

Dans la suite, les anneaux sont des anneaux à unité et les modules sont des modules à gauche unitaires. On dit qu'un anneau $A$ est noethérien (resp. artinien...) si $A$ est un anneau noethérien à gauche (resp. artinien à gauche ...). On note Mod $A$ la eatégorie des $A$-modules à gauche, et $E_{A}(M)$ ou $E(M)$ l'enveloppe injective d'un $A$-mole $M$. La notion duale de la notion d'enveloppe injective est celle de couverture projective et on dit qu'un anneau $A$ est parfait (resp. semi-parfait) si tout $A$-module (resp. tout $A$-module de type fini) possède une couverture projective [1].

Soient $X$ une partie de $A$ et $Y$ une partie d'un $A$-module $M$, on pose $\mathfrak{l}_{A}(Y)=$ $=\{a \in A: a Y=0\}$ et $r_{M}(X)=\{x \in M: X x=0\}$. Si cela n'introduit pas d'ambiguité, on note $\mathfrak{I}(Y)$ au lieu de $\mathfrak{I}_{A}(Y)$. On désigne toujours par $\mathcal{R}(A)$ ou $\mathcal{R}$ le radical de Jacobson de l'anneau $A$.

Pour un module $M$ on note $M^{(I)}$ (resp. $M^{I}$ ) la somme directe (resp. le produit) d'une famille de copies de $M$ indexée par un ensemble $I$.

On dit qu'un module $M$ est co-irréductible si l'intersection de deux sous-modules non nuls quelconques de $M$ est toujours non nulle.

\section{2. - Rappels et complements.}

\section{1. - Modules quasi-injectifs.}

Soit $A$ un anneau, un $A$-module $M$ est quasi-injectif si le foncteur $\operatorname{Hom}_{A}(\cdot, M)$ est exact sur les suites exactes de la forme $0 \rightarrow N^{\prime} \rightarrow M \rightarrow N^{\prime \prime} \rightarrow 0$. Par exemple tout module semi-simple est quasi-injectif. Si $M$ est un module quasi-injectif, tout facteur direct de $M$ et toute somme finie de copies de $M$ sont des modules quasiinjectifs $[17]$. 
Plus généralement, pour un $A$-module $M$ soit $i^{-1}(M)$ la classe des $A$-modules $N$ tels que le foncteur $\operatorname{Hom}_{A}(\cdot, M)$ soit exact sur les suites exactes de la forme $0 \rightarrow$ $\rightarrow N^{\prime} \rightarrow N \rightarrow N^{\prime \prime} \rightarrow 0$, et soit $I_{M}$ la famille des idéaux a de $A$ tels que $A / \mathfrak{a} \in i^{-1}(M)$. Alors la sous-catégorie pleine $i^{-1}(M)$ ayant pour objets les modules de $i^{-1}(M)$ est fermée au sens de [13], i.e. est stable par sousobjets, objets quotients et sommes directes quelconques, ([32] proposition 2.1). et la famille $I_{M}$ est topologisante ([13] voir aussi [3] chapitre $2, \S 2$, exercice 16$)$. De plus un module $N \in i^{-1}(M)$ si et seulement si $N$ coïncide avec le sous-module $I_{M} N$ des éléments $x \in N$ tels que $\operatorname{Ann}(x)$ $\in I_{M}$. On a alors la proposition suivante:

2.1.1. Proposition. - Pour un A-module M les conditions suivantes sont équivalentes:

a) $M$ est un $A$-module quasi-injectif;

b) $M$ est stable par les endomorphismes de son enveloppe injective $E(M)$;

c) On a la relation $I_{M} M=M$.

L'équivalence de $a$ ) et $c$ ) résulte de ce qui précède. L'équivalence de $a$ ) et $b$ ) est le théorème 1.1 de [17].

2.1.2. Remarque. - Pour un $A$-module $M$ désignons par $\Omega_{M}$ (resp. $\bar{\Omega}_{M}$ ) l'ensemble des idéaux de $A$ qui contiennent un idéal de la forme $\operatorname{Ann}(x)$ pour $x \in M$ (resp. de la forme $\operatorname{Ann}(X)$ pour une partie finie $X$ de $M$ ). On a $A n n(x) \in \Omega_{M}$ pour tout $x \in M$, donc les relations $\Omega_{M} \subset I_{M}$ et $I_{M} M=M$ sont équivalentes car la famille $I_{M}$ est topologisante. Or il résulte des définitions que la condition $\Omega_{M} \subset I_{M}$ signifie exactement que:

Pour tout idéal $\mathfrak{b}$ de $A$, tout morphisme $f: \mathfrak{b} \rightarrow M$ dont le noyau appartient à $\Omega_{M}$ se prolonge $\dot{a} A$.

Cette dernière condition, équivalente à la quasi-injectivité de $M$ a été trouvée indépendamment par Fuchs et par des méthodes différentes ([11], voir aussi [28]).

\section{2. - Modules L-quasi-injectifs.}

Remarquons d'abord que tout module semi-simple est $\Sigma$-quasi-injectif, ce fait sera souvent utilisé dans la suite.

La proposition suivante résulte de ([22], théorèmes 1.7 et 1.11$)$.

2.2.1. Proposition. - Pour un A-module quasi-injectif $M$ les conditions suivantes sont équivalentes:

a) $M$ est un module $\Sigma$-quasi-injectif;

b) Le module $M^{(N)}$ est quasi-injectif; 
c) Les éléments de $\bar{\Omega}_{M}$, annulateurs de sous-ensembles de $M$ vérifient la condition maximale (Notation de 2.1.2);

d) $M$ est somme directe d'une famille $\left(E_{i}\right)_{i \in I}$ de modules quasi-injectifs indécomposables telle que toute somme dircote finie des $E_{i}$ soit $\Sigma$-quasi-injective.

2.2.2. Corollatre. - Soit $M$ un module $\Sigma$-quasi-injectit, on peut trouver une décomposition de $M$ en somme directe de modules quasi-injectifs indécomposables de la forme: $M=\oplus \oplus_{i \in I} N_{i}^{\left(\gamma_{i}\right)}$ oì pour $i \neq j$ les enveloppes injectives des modules $N_{i}$ et $N_{j}$ ne sont pas isomorphes.

D'après 2.2.1 $M$ se décompose en somme directe de modules quasi-injectifs indécomposables, soient $N$ et $N^{\prime}$ deux modules figurant dans cette décomposition et tels que les enveloppes injectives $E(N)$ et $E\left(N^{\prime}\right)$ soient isomorphes. Comme le module $N \oplus N^{\prime}$ est quasi-injectif, les modules $N$ et $N^{\prime}$ sont isomorphes ([15], proposition 2.4) et on peut done écrire la décomposition de $M$ comme il est dit dans le corollaire.

La proposition suivante montre que sur un anneau noethérien $A$ tout module quasi-jnjectif possède une telle décomposition.

2.2.3. Propostition. - Pour un anneau $A$ les conditions suivantes sont équivalentes:

a) l'anneau $A$ est noethérien;

b) tout A-module quasi-injectif est $\Sigma$-quasi-injectif;

c) toute somme directe de modules injectifs est un module quasi-injectif;

d) tout module injectif est $\Sigma$-injectif;

e) toute somme directe de modules injectifs est un module injectif.

L'équivalence des conditions $a$ ), $b$ ), c) est la proposition 3.1 de [32];

L'équivalence des conditions $b$ ) et $d$ ) est le théorème 2.3 de [12];

L'equivalence de $a$ ) et $b$ ) est la proposition 4.1 de [6].

2.3. - Modules II-quasi-injectifs.

Rappelons d'abord la caractérisation suivante des modules $\Pi$ quasi-injectifs.

2.3.1. Proposition. - Pour un A-module M les conditions suivantes sont équivalentes:

a) $M$ est $I I$ quasi-injectif;

b) $M$ est un $A / \mathbb{I}_{A}(M)$-module injectif;

c) $M=r_{E(M)}\left(\mathfrak{I}_{A}(M)\right)$ oì $E(M)$ est l'enveloppe injective de $M$;

d) $M=r_{E(M)}(\mathfrak{a})$ pour un idéal a de $A$. 
$a) \Leftrightarrow b)$ est la proposition 2.7 de [32] car $M$ est un $A / \mathfrak{r}_{A}(M)$-module injectif si et seulement si $\mathfrak{l}_{-}(M) \in I_{M}$. Cette équivalence a été trouvée indépendamment par FuLLER qui montre aussi que $a) \Leftrightarrow b) \Leftrightarrow d)$ ([12], théorème 1.2). Il est clair que $c) \Leftrightarrow d$ ).

2.3.2. Remarque. - Tout $A$-module $M$ possède une enveloppe quasi-injective qui est le plus petit sous-module quasi-injectif de $E(M)$ contenant $M$. La proposition 2.3.1 montre que pour tout module $M$ le module $r_{E(M)}\left(\mathfrak{l}_{A}(M)\right)$ est le plus petit sous-module $\Pi$-quasi-injectif de $E(M)$ contenant $M$.

Rappelons qu'un $A$-module $M$ est fidèle si $\mathfrak{I}_{A}(M)=0$.

2.3.3. - CoRollaIRE. - Tout module $\Pi$ quasi-injectif et fidele est injectif.

On dit qu'un anneau $A$ est un $Q$-anneau si tout module quasi-injectif est $\Pi$-quasiinjectif.

On a la caractérisation suivante:

2.3.4. - Proposition ([32], Proposition 2.9). - Un anneau $A$ est un Q-anneau si et seulement si pour tout idéal bilatère a de $A$, tout $A / \mathfrak{a}$-module quasi-injectij et fidète est injectif.

Etudions maintenant une notion qui va jouer un rôle important dans l'étude des $Q$-anneaux et qui va nous permettre de définir les $C 1$ anneaux.

\section{4. - Le cogénérateur minimal de Mod $A$.}

Un $A$-module $Q$ est un cogénérateur de $\operatorname{Mod} A$ si et seulement si tout $A$-module $M$ est isomorphe à un sous-module d'un produit de copies de $Q$; ceci équivaut à dire que $Q$ contient une enveloppe injective de chaque type de module simple ([31], exposé 6 , théorème 1.8 ).

Soit $T$ un ensemble de représentants des types de $A$-modules simples et soit $S=\underset{S \in T}{\oplus} S$; le module $E(S)$ est un cogénérateur injectif de Mod $A$ contenu, à un isomorphisme près, dans tout cogénérateur injectif de Mod $A$, ce module est done un cogénérateur injectif minimal, et, par abus de langage on dira que $E(\$)$ est le cogénérateur minimal de Mod $A$. Ce module est défini à un isomorphisme près, et est fidèle, comme d'ailleurs tout cogénérateur.

2.4.1. DÉFINITION. - On dit qu'un anneau $A$ est un 01 anneau si tout sous-module quasi-injectif du cogénérateur minimal $E$ de $\operatorname{Mod} A$ est $\Pi$-quasi-injectił.

D'après 2.3.1 il revient au même de dire que pour tout sous-module quasi-injectif de $E$ on a $N=r_{E(N)}\left(\mathfrak{l}_{A}(N)\right)$.

2.4.2. Remarque. - Soit $A$ un anneau commutatif noethérien, local et complet pour la topologie définie par son idéal maximum $\mathfrak{m}$, et soit $E=E_{A}(A / \mathfrak{m})$ le cogénérateur minimal de Mod $A$; alors, d'après le théorème 4.2 de $[20]$ on a $N=r_{E}\left(\mathfrak{l}_{A}(N)\right)$ 
pour tout sous-module $N$ de $E$, et comme $E=E_{A}(N)$ lorsque $N \neq 0$, ceci montre que $A$ est un $C 1$ anneau.

On verra à la partie III que la dimension de Krull d'un $Q$-annau noethérien commutatif est toujours au plus égale à 1 . Comme il existe des anneaux locaux noethériens et complets de dimension de Krull $>1$, ceci montre qu'il existe des $C 1$ anneaux qui ne sont pas des $Q$-anneaux.

Par ailleurs, il est clair que tout $Q$-anneau est un $C 1$ anneau.

\section{II. - Généralités sur les $\mathrm{CI}$ anneaux et les $Q$-annuaux.}

Dans cette partie après quelques résultats préliminaires sur le cogénérateur minimal on donne quelques propriétés générales des $C 1$ anneaux et des $Q$-anneaux, en particulier des propriétés de stabilités et une première caractérisation des $O 1$ anneaux qui permet de caractériser les $O 1$ anneaux noethériens sans radicaux et les $Q$-anneaux sans radicaux. Ces propriétés font apparaître entre autres certains caractères noethériens des $Q$ anneaux qui sont complétés par les résultats de REvauLt rappelés au n. 4.

\section{1. - Propriétés du cogénérateur minimal.}

Enonçons d'abord deux lemmes utiles pour la suite:

\subsection{Lemme. - Soit $E$ un cogénérateur de Mod $A$,}

a) pour tout idéal a de $A$ on a la relation $\mathfrak{a}=\mathfrak{l}_{A}\left(r_{E}(\mathfrak{a})\right)$;

b) si $E$ est $\Sigma$-injectif, alors $A$ est anneau noethérien.

a) Pour un idéal a de $A$ il existe une injection $f$ de $A / \mathfrak{a}$ dans un produit $E^{I}$ de copies de $\mathrm{E}$. Posons $f(x)=\left(f_{i}(x)\right)_{i \in I}$ et soit $X=\left\{f_{i}(1+\mathfrak{a}), i \in I\right\}$. On vérifie aisément que $\mathfrak{a}=\mathfrak{l}_{A}(X)$, puis que $\mathfrak{a}=\mathfrak{l}_{A}\left(r_{E}(\mathfrak{a})\right)$.

L'assertion $b$ ) résulte immédiatement de $a$ ) et de la proposition 3 page 184 de [9].

1.2. LEMME. - Soient $\mathfrak{p}$ un idéal bilatère de l'anneau $A$ et $F$ un A-module injectif, alors $r_{F}(\mathfrak{p})$ est un $A / \mathfrak{p}$ module injectif, et si $M$ est un sous-module essentiel de $r_{F}(\mathfrak{p})$ l'enveloppe injective du $A / \mathfrak{p}$-module $M$ est $r_{F}(\mathfrak{p})$.

Soit $f$ un morphisme d'un idéal $G / \mathfrak{p}$ dans $r_{F}(\mathfrak{p})$, le composé $G \rightarrow G / \mathfrak{p} \stackrel{\mathfrak{s}}{\rightarrow} r_{F}(\mathfrak{p})$ se prolonge en une application $A$-linéaire $g: A \rightarrow F$ telle que $g(\mathfrak{p})=0$, done $g$ se factorise par un morphisme $v: A / \mathfrak{p} \rightarrow F$ qui prolonge $f$ car $v(A / \mathfrak{p}) \subset r_{F}(\mathfrak{p})$, et $r_{F}(\mathfrak{p})$ est un $A / \mathfrak{p}$-module injectif. Comme les structures de $A$-module et de $A / \mathfrak{p}$-module de $r_{F}(\mathfrak{p})$ coincident, tout sous- $A$-module essentiel de $r_{F}(\mathfrak{p})$ est un sous- $A / \mathfrak{p}$-module essentiel de $r_{F}(\mathfrak{p})$ d'où la seconde assertion. 
1.3. Composante p-PRIMATRE D'UN MODULE. - Soit $\mathfrak{p}$ un idéal bilatère de l'anneau $A$, pour un $A$-module $M$ on note $M_{(\mathfrak{p})}$ le sous-module de $M$ formé des $x \in M$ annulés par une puissance de $\mathfrak{p}$. On dit que $\boldsymbol{M}_{(\mathfrak{p})}$ est la composante $\mathfrak{p}$-primaire de $\boldsymbol{M}$.

Soit $T$ un ensemble de représentants des types de $A$-modules simples, notons $T_{p}$ la partie de $T$ formée des $S \in T$ tels que $p S=0$. On peut déterminer aisément la composante p-primaire du Socle $\delta=\underset{S \in T}{\oplus} S$ du cogénérateur minimal $E$ de Mod $A$; comme l'annulateur d'un module simple est un idéal premier, on vérifie facilement que pour tout module simple $S$ on a $\mathfrak{p} S=0$ si et seulement si il existe $n$ tel que $\mathfrak{p}^{\prime \prime} S=0$. On a done toujours les inclusions

$$
\underset{S \in T_{\mathfrak{p}}}{\oplus} \mathcal{S} \subset r_{\mathcal{S}}(\mathfrak{p}) \subset S_{\{\mathfrak{p}\}}
$$

Soit $x \in \mathrm{S}_{(\mathrm{p})}$, on peut écrire $x=x_{1}+\ldots+x_{n}$ ò̀ les modules $A x_{i}$ sont simples, appartiennent à $T$ et sont annulés par une puissance de $\mathfrak{p}$, done aussi par $\mathfrak{p}$, et les $A x_{i}$ sont contenus dans $\underset{S \in T}{\oplus} S$. Ceci montre que l'on a les égalités suivantes:

$$
\underset{S \in T_{\mathfrak{p}}}{\oplus}=r_{\mathfrak{S}}(\mathfrak{p})=S_{(\mathfrak{p})}
$$

1.4. Proposition. - Soient $\mathfrak{p}$ un idéal bilatère de $A$ et $E$ le cogénérateur minimal de $\operatorname{Mod} A$, alors:

1) le $A / \mathfrak{p}-$ module $E^{\prime}=r_{E}(\mathfrak{p})$ est le cogénérateur minimal de $\operatorname{Mod} A / \mathfrak{p}$;

2) pour tout $n \geqslant 1$ on a $r_{E}\left(\mathfrak{p}^{n}\right)=r_{F}\left(\mathfrak{p}^{n}\right)$ ou $F$ est une enveloppe injective de $\mathbb{S}_{(\mathfrak{p})}$ dans $E$, et on $a \S_{(\mathfrak{p})} \subset E_{(\mathfrak{p})} \subset F$.

1) Soient $T$ et $T_{\mathfrak{p}}$ les ensembles définis en 1.3 , on peut considérer $T_{\mathfrak{p}}$ comme un ensemble de représentants des types de $A / \mathfrak{p}$-modules simples. Soit $\mathbb{S}^{\prime}=\oplus S$, alors $S^{\prime}$ est un sous-module essentiel de $r_{E}(p)$ comme on le vérifie aisément et la conclusion résulte de 1.2 .

2) On a $E_{(\mathfrak{p})}=\bigcup_{n \geqslant 1} r_{E}\left(\mathfrak{p}^{n}\right)$ par définition, et il suffit de montrer que pour tout entier $n \geqslant 1$ cn a $r_{E}\left(\mathfrak{p}^{n}\right) \subset F$, autrement dit, il suffit de montrer que si $x \in E$ est annulé par $\mathfrak{p}^{n}$ alors $x \in F$. Soit done $x$ tel que $\mathfrak{p}^{n} x=0$, soit $G$ un supplémentaire de $F$ dans $E$, on a $x=y+z$ avec $y \in F$ et $z \in G$, de plus comme la somme $F \oplus G$ est directe on a anssi $\mathfrak{p}^{n} z=0$. Si $z \neq 0$ il existe $a \in A$ tel que $a z \in S$ et $a z \neq 0$, comme $\mathfrak{p}^{n}$ est un idéal bilatère on a $\mathfrak{p}^{n} a z=0$ done $a z \in \mathbb{S}_{(\mathfrak{p})} \subset F^{\dagger}$ et $a z \in F \cap G=\{0\}$ ce qui est absurde, done $z=0$ et $x=y \in F$, ce qui achève la démonstration.

1.5. Propositron. - Soit A un $C 1$ anneau de radical $R$. Si $\bigcap_{n \geqslant 1} \mathfrak{R}^{n}=0$ alors le cogénérateur minimal $E$ de $\operatorname{Mod} A$ est un module $\mathcal{R}$-primaire. 
Soit $E^{\prime}=\bigcup_{n \geqslant 1} r_{E}\left(\Re^{n}\right)$, comme les idéaux $\mathfrak{R}^{n}$ sont bilatéres chaque $r_{E}\left(\mathfrak{R}^{n}\right)$ est un sous-module de $E$ et $E^{\prime}$ est un sous-module de $E$, de plus il est aisé de vérifier que $E^{\prime}$ est stable par tous les endomorphismes de $E$. On a $R S=0$ où $S$ est le socle de $E$ done $\delta \subset E^{\prime}$ et $E^{\prime}$ est essentiel dans $E$ done $E=E_{A}\left(E^{\prime}\right)$ et il résulte de T.2.1.1 que $E^{\prime}$ est un sous-module quasi-injectif de E. D'après le lemme 1.1 on a $\Upsilon_{A}\left(E^{\prime}\right)=$ $=\bigcap_{n \geqslant 1} \mathfrak{l}_{A}\left(r_{E}\left(\mathfrak{R}^{n}\right)\right)=\bigcap_{n \geqslant 1} \Re^{n}$, et $E^{\prime}$ est un module fidèle. Comme $A$ est un $C 1$ anneau, le sous-module quasi-injectif et fidèle $E^{\prime}$ de $E$ est injectif et on a $E^{\prime}=E_{A}\left(E^{\prime}\right)=E$, d'où la proposition.

\section{2. - Premieres propriétés.}

Les propositions suivantes donnent des propriétés de stabilité des $O 1$ anneaux et des $Q$-anneaux, ainsi que des exemples de tels anneaux.

\subsection{Proposition.}

a) Tout anneau artinien est un Q-anneau;

b) Tout produit fini de Q-anneaux (resp. de C1 anneaux) est un Q-anneau, (resp. un 01 anneau).

L'assertion a) est la proposition 2.8 de [32], elle a été trouvée indépendamment par FuluER ([12], corollaire 1.3).

Montrons $b$ ). Soit $A$ un annean produit d'une famille finie d'anneaux $\left(A_{i}\right)_{i \leqslant i \leqslant n}$, on peut écrire $A=\bigoplus_{i=1}^{n} A e_{i}$ où $e_{1}, \ldots, e_{n}$ sont des idempotents centraux et orthogonaux de $A$ tels que pour $1 \leqslant i \leqslant n A_{i}$ soit isomorphe à $A e_{i}$. Soit $M$ un $A$-module, on peut écrire $M=\bigoplus_{i=1}^{n} e_{i} M$ et il est facile de vérifier que $M$ est un $A$-module $I I$-quasiinjectif si et seulement si pour $1 \leqslant i \leqslant n$, le $A e_{i}$-module $e_{i} M$ est $I I$-quasi-injectif. Ainsi $A$ est un $Q$-anneau si et seulement si, pour $1 \leqslant i \leqslant n, A e_{i}=A_{i}$ est un $Q$-anneau.

Il est également facile de vérifier que si $E$ est le cogénérateur minimal de Mod $A$ alors pour $1 \leqslant i \leqslant n e_{i} E$ est le cogénérateur minimal de Mod $A e_{i}$. Comme tout sousmodule quasi-injectif $M$ de $E$ s'écrit $M=\bigoplus_{i=1}^{n} e_{i} M$ où $e_{i} M$ est un sous-module quasiinjectif de $e_{i} E$, l'anneau $A$ est un $O 1$ anneau dès que, pour $1 \leqslant i \leqslant n, A e_{i}=A_{i}$ est un $C 1$ annean.

2.2. Remarque. - En général un prođuit infini de $Q$-anneaux n'est pas un $Q$ anneau et ce n'est pas un $C 1$ anneau comme le montre l'exemple suivant: soit $\left(K_{i}\right)_{i \in I}$ une famille infinie de corps et soit $A$ l'anneau $\prod_{I} K_{i}$, le module $M=\underset{I}{\oplus} K_{i}$ est le socle du $A$-module $A_{s}$ et $M$ est somme d'un $A$-module simple de chaque type. La proposition 6.2 de [26] montre que l'anneau $A$ est auto-injectif et ainsi le $A$-module $A_{s}$ 
est le cogénérateur minimal de Mod $A$. Le $A$-module $M$ est quasi-injectif et fidèle mais n'est pas injectif car $E_{A}(M)=A_{s}$, done, d'après $\mathrm{I}, 2.3 .4, A$ n'est pas un $C 1$ anneau.

2.3. LeMve. - Soient $A$ et $B$ deux anneaux et $T: \operatorname{Mod} B \rightarrow \operatorname{Mod} A$ un foncteur additif. Considérons les propriétés suivantes:

a) $T$ est pleinement fidète et possède un adjoint à gauche $U: \operatorname{Mod} A \rightarrow \operatorname{Mod} B$;

b) l'image par $U$ de toute injection $N \rightarrow T(M)$ ou $M$ est un $B$-module, est une injection;

c) l'image par $T$ d'un monomorphisme essentiel est un monomorphisme essentiel.

d) pour tout B-module simple $S$ le $A$-module $T(S)$ est simple:

1) si $A$ est un Q-anneau et si les propriétés a) b) sont vérifiées, alors $B$ est un Q-anneau;

2) si $A$ est un $C 1$ anneau et si les propriétés a), b), o), d) sont vérifiées alors $B$ est un C1 anneau.

Pour tout $A$-module $N$ et tout $B$-module $M$ on a des morphismes canoniques $\varphi_{N}: N \rightarrow T U(N)$ et $\psi_{M}: U T(\boldsymbol{M}) \rightarrow M$ qui résultent de l'adjonetion de $T$ et $U$.

1) Supposons vérifiées les conditions $a$ ) et $b$ ).

Comme $T$ possède un adjoint à gauche $T$ est exact à gauche et préserve les monomorphismes. Comme $T$ est pleinement fidèle $\psi_{M}$ est un isomorphisme pour tout $B$-module $M$. Montrons qu'un $B$-module $M$ est $\Pi$-quasi-injectif si et seulement si $T(M)$ est un $A$-module $\Pi$-quasi-injectif.

Soit $i: N \rightarrow T(M)$ un sous- $A$-module de $T(M)$ où $M$ est un $B$-module. L'application $\psi_{M} \circ U(i): U(N) \rightarrow M$ est une injection. On a un diagramme commutatif:

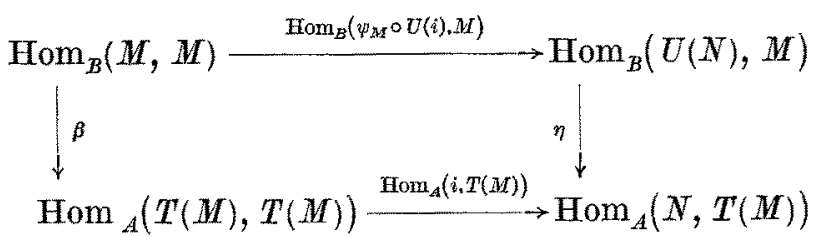

dans lequel $\eta$ est l'isomorphisme de l'adjonction, $\beta$ est l'application définie par $\beta(f)=$ $=T(f)$ et $\beta$ est un isomorphisme car $T$ est pleinement fidèle. Il est facile de vérifier avec ce diagramme que $M$ est quasi-injectif si et seulement si $T(M)$ est quasi-injectif. Comme $T$ possède un adjoint à gauche, $T$ préserve les produits et pour tout $B$-module $M$ et tout ensemble $I$ on a $T\left(M^{I}\right) \rightarrow T(M)^{I}$, ce qui montre que $M$ est $I T$-quasiinjectif si et seulement si $T(M)$ est $\Pi$-quasi-injectif.

Il résulte de ceci que si $A$ est un $Q$-anneau, alors $B$ est un $Q$-anneau.

4-Annali di Matematica 
2) Supposons de plus que $e$ ) soit vérifiée. Soit $M$ un sous- $B$-module quasiinjectif du cogénérateur minimal de $\operatorname{Mod} B$. Le socle $S$ de $M$ est essentiel dans $M$ et les composantes isotypiques de $S$ sont simples. Il est facile de vérifier que si $S$ et $S^{\prime}$ sont des $B$-modules simples non isomorphes, alors $T(S)$ et $T\left(S^{\prime}\right)$ sont des $A$ modules simples non isomorphes et il résulte de $c$ ) que $T(\$)$ est un $A$-module semisimple essentiel dans $T(M)$ dont les composantes isotypiques sont simples. Le module $T(M)$ est quasi-injectif d'après 1 ) et ainsi $T(M)$ est isomorphe à un sous-module quasi-injectif du cogénérateur minimal de $\operatorname{Mod} A$. Si $A$ est un $C 1$ anneau, alors $T(M)$ est $\Pi$-quasi-injectif et aussi $M$ d'après 1 ), done, si $A$ est un $C 1$ anneau, $B$ est un $C 1$ anneau.

2.4. Conollatre. - Soit $f: A \rightarrow B$ un épimorphisme d'anneaux vérifiant l'une des conditions suivantes:

(i) $f$ est surjectif;

(ii) $B$ est un A-module à droite plat;

1) Si $A$ est un $Q$-anneau alors $B$ est un Q-anneau.

2) Si $A$ est un $C 1$ anneau et si pour tout B-module simple $S$ le A-module $f_{*}(S)$ est simple alors $B$ est un $O 1$ anneau. (Condition toujours vérifiée si $f$ est surjectif).

Soit $f_{*}: \operatorname{Mod} B \rightarrow \operatorname{Mod} A$ le foncteur restriction des sealaires et soit $f^{*}=B \otimes_{A}$. Le foncteur extension des scalaires, adjoint à gauche à $f_{*}$. Il résulte de la proposition 2.1 de [24] que $f_{*}$ est pleinement fidèle. Pour un $A$-module $N$ et un $B$-module $M$ on note $\varphi_{N}: N \rightarrow f_{*} f^{*}(N)$ et $\psi_{M}: f^{*} f_{*}(M) \rightarrow M$ les morphismes canoniques. Pour tout $B$-module $M$ le morphisme $\psi_{M}$ est un isomorphisme done aussi $\varphi_{f_{*}(M)}$ car on a la relation $f_{*}\left(\psi_{M}\right) \circ \varphi_{f_{*}(M)}=\mathbf{1}_{f_{*}(M)}$.

Il est facile de vérifier que chacune des conditions (i) et (ii) implique la condition $b$ ) du lemme 2.3 et l'assertion du corollaire relative aux $Q$-anneaux résulte directement du lemme.

Pour terminer la démonstration il suffit de montrer que chacune des conditions (i) et (ii) implique la condition $c$ ) du lemme. La vérification (i) $\Rightarrow c$ ) est immédiate. Montrons que (ii) $\Rightarrow c$ ).

Supposons que $B$ soit un $A$-module à droite plat, alors $f^{*}$ est exact. Soit $i: M \rightarrow P$ un sous- $B$-module essentiel d'un $B$-module $P$. Pour montrer que le monomorphisme $f_{*}(i)$ est essentiel on montre qu'un morphisme $u: f_{*}(P) \rightarrow N$ est injectif dès que $u \circ f_{*}(i)$ est injectif. Si $u \circ f_{*}(i)$ est injectif alors $f^{*}(u) \circ f_{*} f_{*}(i)$ l'est aussi. Comme $\psi_{M}$ et $\psi_{p}$ sont des isomorphismes, l'essentialité de i montre aisément que $f^{*}(u)$ est injectif, done $f_{*} f^{*}(u)$ est aussi injectif; et la relation $f_{*} f^{*}(u) \circ \varphi_{f_{*}(P)}=\varphi_{N} \circ u$ montre que $u$ est injectif car $\varphi_{f_{*}(P)}$ est un isomorphisme. Ceci termine la démonstration.

2.5. CorollaIre. - Soit A un Q-anneau (resp. un C1 anneau), si $P$ est un générateur projectif de type fini alors l'anneau $\operatorname{End}_{A}(P)$ est un $Q$-anneau à droite (resp. un $C 1$ anneau à droite). 
Si $P$ est un générateur projectif de type fini, le foncteur $T=\operatorname{Hom}_{A}(P, \cdot)$ est une équivalence entre Mod $A$ et la catégorie des modules à droite sur l'anneau $T(P)=$ $=\operatorname{End}_{A}(P)$, done $T$ est une équivalence entre $\operatorname{Mod} A$ et la catégorie des modules à gauche sur l'annean opposé à $\operatorname{End}_{A}(P)$. Comme $T$ vérifie les conditions $\left.\left.\left.\left.a\right), b\right), c\right), d\right)$ du lemme on a bien le résultat annoncé.

Suivant B. Roux [30] disons qu'un module simple $S$ est attaché à un module $M$ s'il existe denx sous-modules $N^{\prime}, N$ de $M, N^{\prime} \subset N$ tels que $N / N^{\prime} \simeq S$. On a alors la proposition suivante:

2.6. Propostrion. - Soit $A$ le cogénérateur minimal de Mod $A$,

a) Si $A$ est un $C 1$ anneau alors $A$ vérifie la condition suivante:

(i) Les correspondances $\mathfrak{a} \rightarrow r_{E}(\mathfrak{a})$ et $N \rightarrow \mathfrak{l}_{A}(N)$ sont des bijections décrois santes et inverses l'une de l'autre entre l'ensemble des idéaux bilatères de $A$ contenus dans $\mathcal{R}(A)$ et l'ensemble des sous-modules quasi-injectifs et essentiels de $E$;

b) Considérons les conditions suivantes:

(ii) Tout module simple $S$ est le seul module simple attaché à $E_{A}(S)$;

(iii) Le module $\underset{S \in T}{\oplus} E(S)$, où $T$ est un ensemble de représentants des types de A-modules simples, est injectif.

Si $A$ vérifie les conditions (i), (ii) et (iii) alors $A$ est un $C_{1}$ anneau.

Rappelons qu'un sous-module $N$ d'un module $M$ est caractéristique dans $M$ si $N$ est stable par les endomorphismes de $M$. Il est facile de vérifier que tout sous-module caractéristique d'un module quasi-injectif est quasi-injectif.

a) Soit $N$ un sous-module quasi-injectif et essentiel de $E$, on a $E=E_{A}(N)$ et d'après I.2.3.1, on a $N=r_{E}\left(\mathfrak{l}_{A}(N)\right)$; de plus le socle $\mathcal{S}$ de $E$ est contenu dans $N$ et on $\mathbf{a} \mathfrak{I}_{A}(N) \subset \mathfrak{I}_{A}(S)=\Re(A)$. Inversement, pour un idéal bilatère $\mathfrak{a} \subset \mathcal{R}(A)$ on a $S \subset r_{E}(\mathfrak{R}(A)) \subset r_{E}(\mathfrak{a})$ et $r_{E}(\mathfrak{a})$ est un sous-mođule essentiel de $E$; on vérifle aisément que $r_{E}(\mathfrak{a})$ est un sous-module caractéristique de $E$ et ainsi $r_{E}(\mathfrak{a})$ est quasi-injectif, on a enfin $\mathfrak{a}=\mathfrak{l}_{A}\left(r_{E}(\mathfrak{a})\right)$ avee 1.1 et ceci achève la démonstration de $\left.a\right)$.

b) On conserve les notations de 1.3 Supposons vérifiée la condition (iii), on a alors $E=\underset{S \in T}{\oplus} E_{A}(S)$. Supposons de plus que la condition (ii) soit vérifiée et montrons que pour tout $S \in T$ le module $E_{A}(S)$ est earactéristique dans $E$. Pour cela il suffit de montrer que pour tout module simple $S^{\prime} \in T$ différent de $S$, tout morphisme $f: E_{A}(S) \rightarrow E_{A}\left(S^{\prime}\right)$ est nul, or s'il existait $f: E_{A}(S) \rightarrow E_{A}\left(S^{\prime}\right)$ non nul on aurait $S^{\prime} \simeq$ $\simeq f^{-1}\left(S^{\prime}\right) / \operatorname{Ker}(f)$ ce qui contredirait (ii) et ainsi $E_{A}(S)$ est caractéristique dans $E$. 
Soit maintenant $N$ un sous-module quasi-injectif de $E$, soit $T^{\prime}$ l'ensemble des types de modules simples qui sont dans le socle de $N$. On a $E_{A}(N)=\underset{S \in T^{\prime}}{\oplus} E_{A}(S)$, comme chaque $E_{A}(S)$ est caractéristique dans $E$, le module $E_{A}(N)$ est caractéristique dans $E$, comme $N$ est caractéristique dans $E_{A}(N)$, ceci montre que $N$ est earactéristique dans $E$. Il est alors facile de vérifier que le module $M=N \oplus \underset{S \in T-T^{\prime}}{\oplus} E_{A}(S)$ est caractéristique dans $E$, done $M$ est quasi-injectif; de plus $M$ est essentiel dans $E$ car $M$ contient le socle de $E$. Si $A$ vérifie la condition i), on a $M=r_{E}\left(\mathfrak{I}_{A}(M)\right)$ et $M$ est $\Pi$ quasi-injectif (I.2.3.1) done $N$ est aussi $\Pi$-quasi-injectif ear on vérifie aisément que tont facteur direct d'un module $\Pi$-quasi-injectif est un module $\Pi$-quasi-injectif. $\Pi$ résulte de ceei que tout sous-module quasi-injeetif de $E$ est $\Pi$-quasi-injectif done $A$ est un $O 1$ anneau.

2.7. Remarques. - 1) Un anneau noethérien commutatif possède toujours la condition ii) de 2.6. En effet; soit $\delta \simeq A / \mathfrak{m}$ un $A$-module simple où $\mathfrak{m}$ est un idéal maximal d'un anneau noethérien commutatif $A$, alors $S$ est attaché à $E_{A}\left(S^{\prime}\right)$. Soient $N^{\prime}$, $N$ des sous-modules de $E_{A}(S)$ tels que $N / N^{\prime}$ soit un $A$-module simple, soit $x \in N-N^{\prime}$, d'après le théorème 3.4 de [20] il existe $n$ tel que $\mathfrak{H n}^{n} x=0$ done $\mathfrak{m}^{n} \subset \operatorname{Ann}\left(N / N^{\prime}\right)$ et l'idéal maximal $\operatorname{Ann}\left(N / N^{\prime}\right)$ coïneide avec $\mathfrak{m}$, ceci montre que $N / N^{\prime}$ est isomorphe à $S$ et $S$ est l'unique module simple attaché à $E_{A}(S)$.

2) La condition iii) de 2.6 est en particulier vérifiée dès que $A$ est noethérien, ou dès que l'ensemble des types de modules simples est fini.

\section{3. - $C 1$ anneaux et $Q$-anneaux sans radicaux.}

Rappelons qu'on dit qu'un anneau $A$ est un $V$-anneau si $A$ vérifie les conditions équivalentes suivantes ([10], page 130):

a) Tout module simple est injectif;

b) Tout idéal est l'intersection des idéaux maximaux le contenant.

Dans un tel anneau $\mathcal{O}$ est l'intersection de tous les idéaux maximaux et le radical est nul.

Comme dans 1.3, on désigne par $T$ un ensemble de représentants des types de $A$-modules simples et on pose $\mathcal{S}=\underset{S \in T}{\oplus} S$.

Le lemme suivant montre la relation qui existe entre les $V$-anneaux et les $O 1$ anneaux sans radicaux.

3.1. LEMME. - Les assertions suivantes sont équivalentes:

a) $A$ est un $C 1$ anneau sans radical;

b) le module $\mathrm{S}=\underset{S \in T}{\oplus} S$ est injectif ( $A$ est alors un V-anneau). 
$a) \Rightarrow b)$. On a $E=E(\delta)$ où $E$ est le cogénérateur minimal et d'après $1.1 \mathrm{~S}=$ $=r_{E}\left(\mathfrak{l}_{A}(\delta)\right)$. Comme $\mathfrak{l}_{A}(\delta)=R(A)=0$ on a $\delta=E$ et $\delta$ est injectif. Il est clair que $A$ est un $V$-anneau dès que $S$ est injectif.

$b) \Rightarrow a)$. Si $\delta$ est injectif, alors $A$ est un $V$-anneau et $R(A)=0$. De plus on a alors $E=\delta$ et tout sous-module de $E$ est facteur direct de $E$, donc tout sous-module de $E$ est injectif et à fortiori $I I$-quasi-injectif, ainsi $A$ est un $O 1$ annean.

Les deux lemmes et la proposition suivants nous ont été communiqués par G. ReNaULT. Ils donnent une première décomposition des $C 1$ anneaux sans radicaux.

3.2. LEMvEs. - Le centre $C$ d'un V-anneau $A$ est anneau régulier (au sens de $V$. NEUMANY).

1) Montrons d'abord que pour tout idéal $\mathfrak{a}$ de $A$ on as $\mathfrak{a}=\mathfrak{a}^{2}$. Si $\mathfrak{a} \neq \mathfrak{a}^{2}$ il existe un idéal maximal $\mathfrak{m}$ tel que $\mathfrak{a}^{z} \subset \mathfrak{m}$ et $\mathfrak{a} \dot{4} \mathfrak{m}$. On a alors $1=a+m$ où $a \in \mathfrak{a}$ et $m \in \mathfrak{m}$ d'où $a=a^{3}+a m \in \mathfrak{m}$ et $1=a+m \in \mathfrak{m}$ ce qui est absurde, done $\mathfrak{a}=\mathfrak{a}^{2}$.

2) Soit $C$ le centre de $A$, on a $A x=(A x)^{2}$ pour $x \in C$, done il existe $a \in A$ tel que $x=x^{2} a$. On a alors également $x=x^{2} a=x^{2} a(x a)=x^{2} a(x a)^{2}=x\left(x^{2} a^{3}\right) x$, et tout revient à montrer que $x^{2} a^{3} \in C$. On termine maintenant la démonstration en montrant par récurrence sur $n$ que $x^{2} a^{\text {th }} \in C$ pour tout $n \geqslant 1$. On a $x^{3} a=x \in C$, supposons que $x^{2} a^{n-1} \in O$ et soit $y \in A$, les relations suivantes:

$$
y x^{2} a^{n}=y x^{2} a^{n-1} a=x^{2} a^{n-1} y a=a^{n-1} y x^{2} a=x^{2} a^{n} y,
$$

montrent que $x^{2} a^{n} \in C$ et ceci termine la démonstration.

3.3. Lemme. - Soit $A$ un anneau. Si $\delta=\bigoplus_{S \in T} S$ est un module injectif, alors $A$ ne contient pas une infinité didempotents centraux deus a deux orthogonaux.

Soit $\left(e_{i}\right)_{i \in I}$ une famille d'idempotents centraux deux a deux orthogonaux. Pour tout $i \in I$ soit $\mathrm{m}_{i}$ un idéal à gauche maximal contenant $A\left(1-e_{i}\right)$.

1) Montrons que pour $i \neq j$ les modules simples $A / \mathfrak{m}_{i}$ et $A / \mathfrak{m}_{j}$ ne sont pas isomorphes.

Supposons qu'il existe un morphisme non nul $u: A / \mathfrak{m}_{i} \rightarrow A / \mathfrak{m}_{j}$, alors l'application composée $v: A e_{i} \hookrightarrow A \rightarrow A / \mathfrak{m}_{i} \stackrel{u}{\rightarrow} A / \mathrm{m}_{j}$ est non nulle et est de la forme $a e_{i} \rightarrow$ $\rightarrow a e_{i} x$ pour $x \in A / \mathfrak{m}_{j}$ car $A / \mathfrak{m}_{j}$ est injectif. Posons $x=b+\mathfrak{m}_{j}$ on $a b-e_{j} b=$ $=\left(1-e_{j}\right) b \in \mathfrak{m}_{j}$ et $x=e_{j} b+\mathfrak{m}_{j}=e_{j} x$. Pour tout $a \in A$ on a done $a e_{i} x=a e_{i} e_{j} x=0$ et $v$ est nulle, ce qui estabsurde. Les modules $A / \mathfrak{n t}_{i}$ et $A / \mathfrak{m}_{j}$ sont done non isomorphes.

2) Pour tout $i \in I$ soit $f_{i}: A e_{i} \rightarrow A / \mathfrak{m}_{i}$ l'application définie par $f\left(e_{i}\right)=1+\mathfrak{m}_{i}$. D'après 1) on peut considérer $\underset{I}{\oplus} A / \mathrm{m}_{i}$ comme un sous-module de $S$ et $f=\underset{I}{\oplus} f_{i}$ définit une application de $\underset{r}{\oplus} A e_{i}$ dans $S$. 
Par hypothèse $\underset{I}{\oplus} A e_{i}$ est un idéal de $A$ et $S$ est un $A$-module injectif done $f$ se prolonge en une application $g$ de $A$ dans. $\delta$. Comme $A$ est de type fini Im $g$ est un sous-module de longueur finie de $\mathrm{S}$, done $\underset{I}{\oplus} A / \mathfrak{m}_{i}=\operatorname{Im} f \subset \operatorname{Im} g$ est aussi un module de longueur finie et ceci prouve que $I$ est fini. 3.4. Proposition. - Soit $A$ un 01 anneau sans radical, alors $A=\prod_{i=1}^{n} V_{i}$ où $V_{i}$
est un C1 anneau sans radical dont le centre est un corps.

Soit $A$ un $O 1$ anneau sans radical, le centre $C$ de $A$ est un anneau régulier (3.2) qui ne contient pas une infinité d'idempotents centraux deux à deux orthogonaux (3.1 et 3.3) done $C$ est un anneau semi-simple commutatif, c'est-à-dire $C$ est un produit fini de corps. On peut done écrire $C=C e_{1} \oplus \ldots \oplus C e_{n}$ où $e_{1}, \ldots, e_{n}$ sont des idempotents centraux et deux à deux orthogonaux de $A$, et où $C e_{1}, \ldots, C e_{n}$ sont des corps. On a de plus dans $C$ (done aussi dans $A$ ) la relation $1=e_{1}+\ldots+e_{n}$. Il résulte de ceci que $A=A e_{1} \oplus \ldots \oplus A e_{n}$ et $A$ est isomorphe au produit des anneaux $A e_{1}$, $\ldots, A e_{n}$. Chaque $A e_{i}$ a un radical nul car $\mathcal{O}=\mathfrak{R}(A)=\mathfrak{R}\left(A e_{1}\right) \times \ldots \times \mathfrak{R}\left(A e_{n}\right)$, et chaque $A e_{i}$ est un $C 1$ anneau d'après 2.4 , comme $C e_{i}$ est le centre de $A e_{i}$ il suffit de poser $V_{i}=A e_{i}$ pour avoir le résultat de la proposition.

Nous allons maintenant supposer que $A$ est noethérien, nous pourrons alors avoir des résultats plus précis sur les $V_{i}$ dans ce cas. Commençons par un lemme.

3.5. LEMME. - Soit $A$ un $C 1$ anneau noethérien, alors tout module quasi-injectif dont le socle est essentiel est un module II-quasi-injectif.

a) Montrons d'abord que si $A$ est noethérien, alors toute somme de copies d'un module $\Pi$-quasi-injectif est un module $\Pi$-quasi-injectif.

Si $N$ est $\Pi$-quasi-injectif, on a $N=r_{E(N)}(I(N))$ d'après 1.2 .3 .1 , comme $A$ est noethérien on a $E(N)^{(X)}=E\left(N^{(X)}\right)$ pour tout ensemble $X$ et ainsi on a les relations:

$$
N^{(X)}=r_{E(N)(x)}(\mathfrak{l}(N))=r_{E\left(N^{(x)}\right)}(\mathfrak{l}(N)) .
$$

Comme $\mathfrak{I}(N)=\mathfrak{I}\left(N^{(X)}\right)$ ceci montre que le module $N^{(X)}$ est $\Pi$-quasi-injectif d'après I.2.3.1.

b) Soit maintenant $N$ un module quasi-injectif dont le socle $U$ est essentiel. Comme $A$ est noethérien, il résulte de I.2.2.2 et de I.2.2.3 que $N$ s'écrit $N=\oplus N_{i}^{\left(\gamma_{i}\right)}$ où chaque $N_{i}$ est quasi-injectif indécomposable et où pour $i \neq j$ les modules $E\left(N_{i}\right)$ et $E\left(N_{i}\right)$ ne sont pas isomorphes. Pour $i \in I$ le module $N_{i}$ est quasi-injectif indécomposable done $N_{i}$ est coirréductible, comme $U$ est essentiel dans $N$ le socle $U \cap N_{i}$ de $N_{i}$ est non nul et ce socle est done simple, soit $S_{i}$ le socle simple de $N_{i}$. Comme $E\left(S_{i}\right)=E\left(N_{i}\right)$ les modules $S_{i}$ et $S_{j}$ ne sont pas isomorphes pour $i \neq j$ et le module $\oplus E\left(N_{i}\right)$ est isomorphe à un sous-module du cogénérateur minimal $E$ de $\operatorname{Mod} A$. $i \in I$

Soit $M=\underset{i \in I}{\oplus} N_{i}$, le module $M$ est quasi-injectif car $M$ est facteur direct de $N$ comme 
on le vérifie aisément et $M$ est done isomorphe à un sous-module quasi-injectif de $E$ Comme $A$ est un $O 1$ anneau, $M$ est $\Pi$-quasi-injectif. Soit $g=\sum_{i \in I} \gamma_{i}$ et soit $K_{i}=$ $=\mathfrak{\gamma}-\check{\gamma}_{i} ;$ pour $i \in I$, on a $M^{(\mathfrak{f})}=N \oplus \oplus_{i \in I} N_{i}^{\left(K_{i}\right)}$ et $N$ est facteur direct de $M^{(\mathscr{(})}$; d'après $a$ ) le module $M^{(\gamma)}$ est $\Pi$-quasi-injectif et $N$ est $\Pi$-quasi-injectif comme facteur direct d'un module $\Pi$-quasi-injectif.

3.6. Thtónème. - Pour un anneau A les conditions suivantes sont équivalentes:

a) A est un 01 anneau noethérien sans radical;

b) $A$ est un $V$-anneau noethérien;

c) A est produit fini de V-anneaux noethériens quasi-simples;

d) tout A-module semi-simple est injectif.

$a) \Rightarrow d$ ). Soit toujours $S=\underset{S \in T}{\oplus} S$. Le module $S$ est fidèle car $A$ est sans radical. Soit $U$ un module semi-simple, alors $U \oplus S$ est semi-simple donc $\Pi$-quasi-injectif d'après 3.5 , or $U \oplus S$ est fidèle dono $U \oplus S$ est injectif d'après I.2.3.3 et $U$ est injectif.

$d) \Rightarrow b$ ). Si $d$ ) est vérifié il est clair que $A$ est un $V$-anneau. Le module $\delta$ est injectif done coïncide avec le cogénérateur minimal $E=E(\delta)$ de $\operatorname{Mod} A$. Le module $\delta$ est donc un cogénérateur $\Sigma$-injectif et $A$ est noethérien d'après 1.1.

$b) \Rightarrow c)$. Le radical de $A$ est nul done $A$ est semi-premier et il résulte de [10] (page 130 ) que le $V$-anneau noethérien $A$ est produit fini de $V$-anneaux noethériens quasi-simples.

$e) \Rightarrow a$ ). Un produit fini de $V$-anneaux noethériens quasi-simples est un anneau noethérien sans radical, et d'après 2.1 il suffit de montrer qu'un $V$-anneau noethérien est un 01 anneau mais ceci résulte immédiatement de 3.1 et le théorème est donc démontré.

L'équivalence $b) \Leftrightarrow d$ ) a été trouvée indépendament et par une néthode différente par K. A. Bxad ([5], proposition 1).

\section{REMARQUES:}

1) Soit $A$ un $V$-anneau noethérien quasi-simple, alors $A$ est un $C 1$ anneau quasi-simple done sans idéaux bilatères $\neq 0$ et d'après 3.4 le centre de $A$ est un corps.

2) Il résulte aisément de 3.4 et 3.6 que si $A$ est un $V$-anneau noethérien alors $A$ est quasi-simple si et seulement si le centre de $A$ est un corps.

3.7. ThÉoRème. - Pour un anneau A les conditions suivantes sont équivalentes:

a) A est un Q-anneau sans radical;

b) A est un Q-anneau noethérien sans radical; 
c) A est produit fini de $V$-anneaux noethériens quasi-simples $\left(B_{i}\right)_{1 \leqslant i \leqslant n}$ tels que tout $B_{i}$-module quasi-injectif est injectif;

d) tout A-module quasi-injectif est injectif.

Les implications $a) \Rightarrow b$ ) et $b$ ) $\Rightarrow c$ ) résultent de la proposition 1 et du lemme 2 de $[27]$.

$c) \Rightarrow d)$. Soit $A=\prod_{i=1}^{n} B_{i}$, on peut écrire $A=\bigoplus_{i=1}^{n} A e_{i}$, où les $e_{i} 1 \leqslant i \leqslant n$ sont des idempotents centraux et orthogonaux de $A$ tels que pour $1 \leqslant i \leqslant n$ les anneaux $A e_{i}$ et $B_{i}$ sont isomorphes, done tout $A e_{i}$-module quasi-injectif est injectif. Soit $M$ un $A$-module quasi-injectif, montrons que $M$ est injectif. On peut ecrire $M=\bigoplus_{i=1}^{n} e_{i} M$, pour $1 \leqslant i \leqslant n e_{i} M$ est un $A$-module quasi-injectif comme facteur direct de $M$ et $e_{i} M$ est aussi un $A e_{i}$-module quasi-injectif donc $e_{i} M$ est un $A e_{i}$-module injectif et on vérifie alors aisément que $M$ est un $A$-module injectif.

d) $\Rightarrow a)$. Si tout $A$-module quasi-injectif est injectif, alors $A$ est un $Q$-anneau et un $V$-anneau et un $V$-amneau est toujours sans radical.

3.8. Remarque. - Donnons un exemple de $V$-anneau noethérien quasi-simple tel que tout module quasi-injectif soit injectif. Cet exemple est dù à G. Renault ([31], exposé 20) et utilise un anneau introduit par J. H. Cozzens [7].

Soit $Q$ la clôture algébrique du corps $\boldsymbol{Z} / 2 \boldsymbol{Z}$ et soit f l'auto-morphisme de $\Omega$ défini par $f(a)=a^{2}$. On définit l'anneau $A=\Omega[X, \varrho]$ comme l'ensemble des polynômes formels $\sum_{i=0}^{n} a_{i} X^{i}$ muni de la relation $X a=a^{2} X$. Soit $S$ l'ensemble multiplicatif formé des $X^{k}, k \geq 0$, on peut construire l'anneau des quotients $A_{S}$ et l'anneau $A_{s}$ possède les propriétés suivantes: ([31], exposé 20, théorème 7 ):

a) $A_{\mathcal{S}}$ est un anneau sans diviseurs de 0 principal à gauche et à droite;

b) $A_{S}$ est un anneau quasi-simple;

c) $A_{S}$ est un $V$-anneau à droite;

d) tous les modules à droite simples sont isomorphes.

Le corollaire 10 de ([31], exposé 20$)$ montre que tout $A_{S}$-module quasi-injectif est un $A_{S}$-module injectif.

3.9. Corollatre. - Pour tout Q-anneau $A$, l'anneau $A / R(A)$ est un V-anneau noethérien.

3.10. CoRolidire. - Un anneau commutatif et sans radical est un Q-anneau si et seulement si il est produit fini de corps. 
Soit $A$ un anneau réduit (i.e. sans élément nilpotent), comme un idéal simple d'un anneau est engendré par un idempotent ou est de carré nul tout idéal simple non nul de $A$ est engendré par un idempotent non nul.

Considérons l'anneau $A_{S}$ de 3.4 , comme $A_{S}$ est un $Q$-anneau sans diviseurs de zéro, c'est un $Q$-anneau réduit et l'unité de $A_{S}$ est l'unique idempotent non nul de $A_{S}$, done $A_{S}$ ne contient aucun idéal propre simple non nul. Comme $A_{S}$ 'est pas un corps, le socle de $A_{S}$ est nul. On a plus généralement le résultat suivant:

3.11. Propositiox. - Un Q-anneau (resp. un O1 anneau) réduit $A$ est produit divect d'un produit fini de corps et d'un Q-anneau (resp. un C1 anneau) réduit à socle nul.

Montrons d'abord la proposition lorsque $A$ est un $C 1$ anneau.

Soit $\&$ un idéal simple du $O 1$ anneau réduit $A$, comme $A$ ne contient pas d'élément nilpotent non nul, on a $S=A e$ où e est un idempotent de $A$, de plus l'exercise 17 du $\$ 6$ de [2] montre que $e$ est central.

On pent done écrire le socle (ganche) $\mathcal{S}$ de $A$ sous la forme $\mathcal{G}=\oplus_{i \in I} A e_{i}$ où les $e_{i}$, $i \in I$ sont des idempotents centraux et orthogonaux de $A$. Comme $A$ est réduit, on a $\mathcal{G} \cap \Re(A)=0$ car si $0 \neq x \in \mathcal{G} \cap \Re(A)$ l'idéal $A x$ contient un idempotent non nul, ce qui est absurde car $\mathcal{R}(A)$ ne contient aucun idempotent non nul. Ceci montre que $\mathcal{G}$ s'injecte dans $A / \mathcal{R}(A)$.

Il rèsulte alors de 3.3 et 3.7 que les $\bar{e}_{i}=e_{i}+R(A)$ sont en nombre fini, done aussi les $e_{i}$, et $e^{\prime}=\sum_{i \in I} e_{i}$ est un idempotent central de $A$. On a alors $A=G \times A\left(1-e^{\prime}\right)$ où $A\left(1-e^{\prime}\right)$ est un $C 1$-anneau rèduit à socle nul et $G=A e^{\prime}$ est un anneau semisimple rèduit done produit fini de corps d'aprés le lemme 6.1 de [29].

Montrons maintenant la proposition lorsque $A$ est un $Q$-anneau réduit. Si $A$ est un $Q$-anneau alors $A$ est un $C 1$ anneau et $A=B \times C$ où $B$ est un produit fini de corps et $C$ est un $C 1$ anneau réduit à socle nul. L'anneau $C$ est isomorphe à un quotient de $A$ et la proposition $2.4 a$ ) montre que $C$ est un $Q$-anneau.

\section{4. - Caracteres noethériens des $Q$-anneaux.}

On a vu en 3.5 qu'un $Q$-anneau sans radical est un anneau noethérien. Les deux théorèmes suivants, dûs à $G$. RENAULt, montrent qu'il existe d'autres conditions sous lesquelles les $Q$-anneaux vérifient des conditions de chaîne.

4.1. ThÉonł̇me ([27], Théorème 5). - Soit $A$ un Q-anneau de radical $\mathcal{R}$ tel que $A / \mathcal{R}$ soit semi-simple, et soit $\mathfrak{a}=\bigcap_{n \geqslant 0} \Re^{n}$. Alors l'anneau $A / \mathfrak{a}$ est un $Q$-anneau noethérien.

4.2. THÉoRÈme ([27], théorème 4). - Un Q-anneau (à gauche) parfait à gauche ou parfait à droite est artinien à gauche.

4.3. ConollaIRE. - Soit $A$ un Q-anneau tel que $A / R$ soit semi-simple, alors pour tout entier n l'anneau $A / \Re^{n}$ est artinien. 
En effet, $A / \Re^{n}$ est un $Q$-anneau d'après 2.4 et est parfait d'après le théorème $P$ de [1].

Rappelons qu'un anneau $A$ est semi-artinien si tout module non nul a un socle essentiel [23]. On a alors:

\subsection{Proposttion. - Un Q-anneau semi-artinien est artinien.}

Soit $A$ un $Q$-anneau semi-artinien, et soit $R$ son radical, il résulte de 2.4 et de la proposition 3.2 de $[23]$ que $A / \mathcal{R}$ est un $Q$-anneau semi-artinien sans radical. Soit $\mathcal{S}$ le socle de $A / R$, l'exercice 4 du $\S 6$ de [2] montre que $S \cap \mathfrak{I}_{A / R}(\$)=0$, comme $\delta$ est essentiel dans $A / \mathcal{R}$, on a $\mathfrak{I}_{A / \mathcal{R}}(\delta)=0$ et le module quasi-injectif $\delta$ est fidèle,

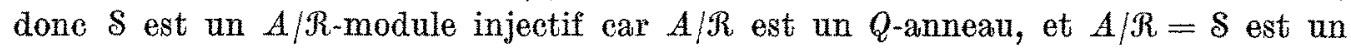
anneau semi-simple. La proposition 3.2 de [23] montre aussi que $\mathcal{R}$ est $T$-nilpotent à droite donc $A$ est un anneau parfait d'après le théorème $P$ de [1] et $A$ est artinien avec 4.2 .

\subsection{Remarques:}

1) Soit $A$ un anneau local commutatif de radical $\mathfrak{m} \neq 0$ tel que $\mathfrak{m}^{2}=0$. Si $A$ n'est pas noethérien, le corollaire 4.3 montre que $A$ n'est pas un $Q$-anneau, done d'après I.2.3.4 il existe un idéal bilatère $\mathfrak{a}$ de $A$ et un $A / \mathfrak{a}$-module quasi-injectif et fidèle qui n'est un $A / \mathfrak{a}$-module injectif. En fait, ici on peut construire explicitement un $A$-module quasi-injectif et fidèle non injectif.

Supposons done que $A$ ne soit pas noethérien. Comme $\mathfrak{m}^{2}=0$ le radical $\mathfrak{m}$ de $A$ est un $A / \mathrm{mt}$-module dono $\mathrm{m}$ est un $A / \mathrm{m}$-module semi-simple et on peut écrire $\mathfrak{m}=\oplus_{i \in I} A a_{i}$ où pour $i \in I \operatorname{Ann}\left(a_{i}\right)=\mathfrak{m}$. Montrons d'abord que $I$ est infini. Si $I$ est fini on peut éerire $I=\left\{i_{1}, \ldots, i_{n}\right\}$ et la suite

$$
0, A a_{1}, A a_{1} \oplus A a_{2}, \ldots, \oplus_{k=1}^{n-1} A a_{i_{k}}, \mathfrak{n t}, A,
$$

est alors une suite de Jordan Holder de $A$, mais ceci est absurde car $A$ n'est pas noethérien, done $I$ est infini.

Soit maintenant $E=E(A / \mathfrak{m})$ et soit $x_{0}=1+\mathfrak{m} \in A / \mathfrak{m}$. Pour $i \in I$ posons $\mathfrak{a}_{i}=$ $=\oplus_{j \neq i} A a_{j}$. Comme $E$ est injectif, l'application linéaire $g_{i}: \mathfrak{m} \rightarrow E$ définie pour $i \in I$ par $g_{i}\left(a_{i}\right)=0$ et $g_{i}\left(a_{i}\right)=x_{0}$ se prolonge en une application linéaire de $A$ dans $E$. Il existe done $x_{i} \in D$ tel que pour tout $a \in \mathfrak{M} g_{i}(a)=a x_{i}$. On a en partieulier $a_{i} x_{i}=x_{0}$ et $\mathfrak{a}_{i} x_{i}=0$. Cette dernière relation montre que $\mathfrak{a}_{i} \subset \operatorname{Ann}\left(x_{i}\right)$. Montrons que $\mathfrak{a}_{i}=\operatorname{Ann}\left(x_{i}\right)$. Soit $a \in \operatorname{Ann}\left(x_{i}\right)$, on a $\operatorname{Ann}\left(x_{i}\right) \subset \mathrm{m}$ car $x_{i} \neq 0$, done $a \in \mathfrak{m}$ et on peut écrire $a=c a_{i}+b$ où $e \in A$ et $b \in \mathfrak{a}_{i}$. Comme $\mathfrak{a}_{i} x_{i}=0$ on a $b x_{i}=0$ et les relations $0=a x_{i}=c a_{i} x_{i}=c x_{0}$ montrent que $e \in \operatorname{Ann}\left(x_{0}\right)=\mathfrak{m} ;$ or $\mathfrak{m}=\operatorname{Ann}\left(a_{i}\right)$ done $c a_{i}=0$ et $a=b \in \mathfrak{a}_{i}$.

Considérons l'application linéaire $g: \mathfrak{m} \rightarrow E$ définie par $g\left(a_{i}\right)=x_{0}$ pour tout $i \in I$. Par injectivité de $E$ cette application se prolonge à $A$ et il existe $z \in E$ tel que pour 
tout $a \in \mathfrak{M} g(a)=a z$, on a done $a_{i} z=x_{0}$ pour tout $i \in I$. Comme $A$ est commutatif $\mathfrak{a}_{i}$ est un idéal bilatère de $A$ pour tout $i$ et il est immédiat de vérifier que $r_{B}\left(\mathfrak{a}_{i}\right)$ est un sous-module caractéristique de $E$ pour tout $i$. Le module $N=\sum_{i \in I} r_{E}\left(a_{i}\right)$ est aussi un sous-module caractéristique de $E$ done $N$ est un sous-module quasi-injectif de $E$.

On va montrer que $N$ est fidèle et n'est pas injectif. Pour $i \in I$ on a $\mathfrak{a}_{i} x_{i}=0$ done $x_{i} \in N$ et $\Upsilon(N) \subset \bigcap_{i \in I} \operatorname{Ann}\left(x_{i}\right)=\bigcap_{i \in I} \mathfrak{a}_{i}$, comme $\bigcap_{i \in I} \mathfrak{a}_{i}=0$ on a $\mathfrak{l}(N)=0$ et $N$ est
fidèle.

Comme $E$ est indécomposable tout sous-module non nul de $E$ est essentiel dans $E$ et $E=E(N)$ et pour montrer que $N$ est non injectif il suffit de montrer que $N \neq E$. Pour cela on montre que $z \notin N$. Raisonnons par l'absurde; si $z \in N$ on peut écrire

$$
z=\sum_{k=1}^{n} y_{k}
$$

où $y_{k} \in r_{E}\left(\mathfrak{a}_{i_{k}}\right)$, comme $I$ est infini, il existe $i \in I$ tel que $i \notin\left\{i_{1}, \ldots, i_{n}\right\}$, on a alors $a_{i} \in \mathfrak{a}_{i_{k}}$ pour $1 \leqslant k \leqslant n$ et $a_{i} z=\sum_{k=1}^{n} a_{i} y_{k}=0$. Ceci est absurde car $a_{i} z=x_{0} \neq 0$.

En résumé, le module $N$ est un module quasi-injectif, fidèle et non injectif.

2) Lorsque $A$ est un $Q$-anneau la condition " $A / \mathcal{R}$ semi-simple 》 du théorème 4.1 n'est pas nécessaire pour que $A / \bigcap_{n \geqslant 1} \Re^{n}$ soit noethérien, par exemple l'anneau $A_{S}$ de 3.4 est un $Q$-anneau sans radical, noethérien et non semi-simple.

Si $A$ est un $Q$-anneau commutatif, la condition $A / R$ semi-simple est toujours vérifiée d'après 3.6 ; cette condition est aussi vérifiée lorsque $A$ est un $Q$-anneau auto-injectif à gauche (i.e. $A$ est un $A$-module à gauche injectif) (cf. [27]).

\section{III - Etude du cas noetherien non commutatif.}

Soit $A$ un $Q$-anneau de radical $R$, alors $A / \mathcal{R}$ est produit fini de $V$-anneaux noethériens quasi-simples (II.3.3). Dans la mesure où les $V$-anneaux sont assez mal connus dans le cas non commutatif, il semble difficile d'avoir des résultats sur la structure de $A$ sans faire des hypothèses supplémentaires portant par exemple sur $A / R$ : par exemple on a vu en II.4.1 que si $A / \mathcal{R}$ est semi-simple, alors $A / \bigcap_{n \geqslant 0} R_{n}$ est un anneau noethérien. On étudie ici la structure de $A / \bigcap_{n \geq 0} R^{n}$ lorsque $A$ est un $C 1$ anneau ou un $Q$-anneau noethérien et lorsque $A / R$ est quasi-simple ou semi-simple $D$ 'après II.2.4, on peut supposer que $\bigcap_{n \geqslant 0} \mathcal{R}_{n}=0$.

Comme la terminologie relative aux anneaux locaux non commutatifs varie becaucoup suivant les auteurs, nous précisons celle que nous utilisons:

On dit qu'un annearu $A$ de radical $R$ est quasi-local (resp. semi-local) si $A$ vérifie 
les conditions suivantes:

1) L'anneau $A$ est noethérien;

2) On a $\bigcap_{n \geqslant 0} \mathfrak{R}^{n}=0$;

3) L'anneau $A / R$ est quasi-simple (resp. $3^{\prime}$ ) l'anneau $A / \Omega$ est semi-simple).

Le condition 3) exprime que $R$ est l'unique idéal bilatère maximal de $A$. Si $A$ est un anneau quasi-local ou semi-local tel que $A / \mathcal{R}$ soit un anneau simple on dit que $A$ est un anneau local.

Dans une première partie on étudie le séparé complété $\hat{A}$ d'un $C 1$ anneau semilocal $A$ pour la topologie $R$-adique et on montre que $\hat{A}$ se comporte à peu près comme un anneau de ZaRIsKI.

Dans une seconde partie on caractérise certains $Q$-anneaux quasi locaux.

Pour un idéal bilatère $\mathfrak{p}$ de $A$ les notations $E, E_{(\mathfrak{p})}, \delta, \delta_{(\mathfrak{p})}$ sont celles de II.1.3.

\section{A) Séparé complété d'un $O 1$ anneau semi-local.}

\section{1. - Preliminaires.}

Soit $A$ un anneau, soit $\mathcal{C}$ une sous-catégorie fermée de Mod $A$, pour un $A$-module $M$ on note $T_{\mathrm{C}} M$ la topologie linéaire sur $M$ ayant pour sous-modules ouverts les sousmodules $N$ de $M$ tels que $M / N \in \mathrm{C}$.

Soit $\mathfrak{p}$ un idéal bilatère de $A$, pour un $A$-module $M$ la topologie linéaire sur $M$ pour laquelle les sous-modules $\mathfrak{p}^{n} M, n \geqslant 0$, sont un système fondamental de voisinages de 0 , est la topologie $\mathfrak{p}$-adique de $M$ qui est notée $T_{\mathfrak{p}} M$.

On note $\hat{A}$ le séparé complété de $A$ pour la topologie p-adique. On a un morphisme canonique d'anneaux $i: A \rightarrow \hat{A}$ dont le noyau est $\bigcap_{n \geqslant 0} \mathfrak{p}^{n}$ et $\hat{A}$ est un anneau séparé et complet pour la topologie linéaire définie par les idéaux $\hat{p^{n}}$ où $\hat{p^{n}}$ est l'adhérence de $i\left(\mathfrak{p}^{n}\right)$ dans $\hat{A}$. On a alors le résultat suivant qui résulte de ([3], chapitre 3 , formules (18), (19), (21) page 49).

1.1. Lemwe. - Pour tout entier $n$ on a $\hat{p}^{n}=i^{-1}\left(\hat{p^{n}}\right)$ et $\hat{A}=i(A) \hat{p^{n}}$ et le morphisme canonique $A / \mathfrak{p}^{n} \rightarrow \hat{A} / \hat{p}^{n}$ est un isomorphisme.

2. - Le complété d'un 01 anneau semi-local.

2.1. DéfintTron. - Soit $\mathfrak{p}$ un idéal bilatère d'un anneau $A$ et soit $\mathrm{C}=\mathrm{Dis} A$ la sous-eatégorie des A-modules discrets associée à la famille topologisante des idéaux qui contiennent une puissance de $\mathfrak{p}$. On dira que A vérifie la condition $R_{\mathfrak{p}}$ si $R_{\mathfrak{p}}$ : Pour tout A-module $M$ et tout sous-module $N$ de $M$ la topologie $T_{\mathrm{C}} N$ est induite par la topologie $T_{\mathrm{C}} M$.

2.2. Proposirton. - Soit $A$ un $C 1$ anneau noethérien tel que pour un idéal bilatère $\mathfrak{p}$ la topologie $\mathfrak{p}$-adique soit artinienne et séparée, alors $A$ vérifie la condition $R$. 
Comme $0=\bigcap_{n \geqslant 0} \mathfrak{p}^{n}$ est l'annulateur de $E_{(\mathfrak{p})}$ le module $E_{(\mathfrak{p})}$ est fidèle, comme $A$ est un $O 1$ anneau, le module quasi-injectif $E_{(\mathfrak{p})}$ est $I I$-quasi-injectif done $E_{(\mathfrak{p})}$ est injectif d'après I.2.3.3. Pour montrer que $R_{\mathfrak{p}}$ est vérifiée il suffit d'après la proposition 9 page 427 de [13] de montrer que $\mathcal{C}$ est stable par enveloppes injectives. Soit $M \in \mathcal{C}$, pour tout $x \in M$ il existe $n$ tel que $\mathfrak{p}^{n} x=0$ et $\mathfrak{p}^{n} A x=0$ car $\mathfrak{p}^{n}$ est bilatère, done $A x$ est un $A / \mathfrak{p}^{n}$-module et le socle de $A x$, comme $A$-module on comme $A / \mathfrak{p}^{n}$-module, n'est pas nul. Ceci montre que le socle $S$ de $M$ est essentiel dans $M$. L'enveloppe injective de $M$ coïncide done avec $E_{A}(S)$. Soit $T^{\prime}$ l'ensemble des types de modules simples qui figurent dans $S$, comme $S$ est discret tout module simple $U \in T^{\prime}$ est discret i.e. annulé par $\mathfrak{p}$ et $U$ est facteur direct de $\mathrm{S}_{(\mathfrak{p})}$ (cf. II.1.3). Posons $S=\underset{U \in T^{\prime}}{\oplus} U^{\left(X_{U}\right)}$, posons $X=\sum_{T^{\prime}} X_{U}$ et soit $Y_{U}=X-X_{U}$ on vérifie aisément que $S_{(p)}^{(X)}$ est somme directe de $S$ et de $\underset{U \in T^{\prime}}{\oplus} U^{\left(Y_{v} v\right)}$. Ceci montre que $E_{A}(S)$ est facteur direct de $E_{A}\left(\delta_{(\mathfrak{p})}^{(X)}\right)$, comme $A$ est noethérien $E_{A}\left(\S_{(\mathfrak{p})}^{(X)}\right)$ est isomorphe à $E_{A}\left(\delta_{(\mathfrak{p})}\right)^{(X)}$ et ainsi $E_{A}(M)=E_{A}(S)$ est facteur direct d'une somme directe de copies de $E_{A}\left(\delta_{(p)}\right)$. Comme $E_{(\mathfrak{p})}$ est injectif, on a $E_{(\mathfrak{p})}=E_{A}\left(\mathrm{~S}_{(\mathfrak{p})}\right)$ d'après II.1.4 et $E_{A}\left(S_{(\mathfrak{p})}\right)=E_{(\mathfrak{p})} \in$ C. La catégorie fermée $\mathcal{C}$ est stable par sommes directes et sous-objet done $D_{A}(M)$ appartient $\grave{a} \mathcal{C}$ et $\mathcal{C}$ est stable par enveloppes injectives, ce qui achève la démonstration.

2.3. LeMMe. - Soit $A$ un anneau noethérien et soit $\mathfrak{p}$ un idéal bilatère de $A$ tel que $\bigcap_{n \geqslant 0} \mathfrak{p}^{n}=0$. si la condition $R_{\mathfrak{p}}$ est vérifiée alors:

a) pour tout $A$-module de type fini $M$ le morphisme canonique $\hat{A} \otimes_{A} M \rightarrow \hat{M}$ où $\hat{M}$ est le complété séparé de $M$ pour la topologie p-adique, est bijectif;

b) le A-module à droite $\hat{A}$ est plat;

c) si $\mathfrak{p}=\Re(A)$ alors tout module de type fini est séparé et $\hat{A}$ est fidèlement plat.

La démonstration du lemme est assez semblable à celle du cas commutatif nous la donnons néanmoins en détail.

Cette démonstration va se faire en plusieurs étapes. Soit toujours e la catégorie fermée définie en 2.1 .

1) Pour tout module de type fini $M$ les topologies $T_{\mathfrak{C}} M$ et $T_{\mathrm{p}} M$ coüncident.

Soit $N$ un sous-module ouvert de $M$ pour $T_{\mathfrak{p}} M$, il existe $n$ tel que $\mathfrak{p}^{n} M \subset N$ et par suite $M / N \in \mathrm{C}$, donc $N$ est ouvert pour $T_{\mathrm{C}} M$. Réciproquement si $M$ est engendré par $x_{1}, \ldots, x_{q}$ et si $N$ est ouvert pour $T_{\mathbb{C}} M$ il existe un entier $n$ tel que $\mathfrak{p}^{n} x_{i} \subset N$ pour $1 \leqslant i \leqslant q$ et ainsi $\mathfrak{p}^{n} M \subset N$ done $N$ est ouvert pour $T_{\mathfrak{p}} M$.

2) Pour tout module de type fini $M$ on a $x \in \bigcap_{n \geqslant 0} \mathfrak{p}^{n} M$ si et seulement si il existe $a \in \mathfrak{p}$ tel que $(1-a) x=0$.

Il est clair que si $x=a x$ avec $a \in \mathfrak{p}$ alors $x=a^{n} x$ pour tout entier $b$ et $x \in \bigcap_{n \geq 0} \mathfrak{p}^{n} M$ 
Réciproquement si $x \in F=\bigcap_{n \geqslant 0} \mathfrak{p}^{n} M$ la topologie $\mathfrak{p}$-adique de $A x$ coïndice avec $T_{\mathrm{C}} A x$ donc est induite par la topologie $T_{\mathrm{C}} M$ qui coïncide avec la topologie p-adique de $M$ ainsi la topologie $\mathfrak{p}$-adique de $A x$ est la topologie grossière et $A x=\mathfrak{p} A x$, en particulier $x=a x$ avec $a \in \mathfrak{p}$.

3) Montrons maintenant que pour toute suite exacte $F \stackrel{n}{\rightarrow} G \stackrel{v}{\rightarrow} H$ de A-modules de type fini, la suite $\hat{F} \stackrel{\hat{u}}{\rightarrow} \hat{G} \stackrel{\hat{v}}{\rightarrow} \widehat{H} q u$ 'on en déduit par passage aux séparés complétés pour les topologies $\mathfrak{p}$-adiques, est exaote.

D'abord $u$ et $v$ sont des morphismes stricts pour les topologies $\mathfrak{p}$-adiques de $F$, $G$ et $H$, en effet, pour $n>0$ les relations $u\left(\mathfrak{p}^{n} F^{n}\right)=\mathfrak{p}^{n} u(F)$ montrent que l'image par $u$ de tout voisinage de 0 dans $F$ pour $T_{\mathfrak{p}} F$ est un voisinage de 0 dans $u(F)$ pour $T_{\mathfrak{p}} u\left(F^{\prime}\right)$, or d'après la condition $R_{\mathfrak{p}}$ et 1$)$ la topologie $T_{\mathfrak{p}} u(F)$ coüncide avec la topologie induite sur $u(F)$ par $T_{\mathfrak{p}} M$, ainsi $u$ est un morphisme strict d'après un résultat de [4] (chap. $3, \S 2$, n. 8 , prop. 2.4.); on montre de la même façon que $v$ est un morphisme strict.

L'exactitude du foncteur complétion p-adique sur les modules de type fini résulte alors d'un lemme de [3] (chap. 3. §2, n. 12, lemme 2).

4) Montrons maintenant que pour tout module de type fini M le morphisme canonique $\alpha_{M}: \hat{A} \otimes_{A} M \rightarrow \hat{M}$ est bijectif.

Cette assertion est évidente si $M=A$ et on se ramène immédiatement à ce cas si $M$ est libre de type fini, done lassertion est aussi vraie pour tout module libre de type fini. Dans le cas général comme $A$ est noethérien et $M$ est de type fini, il existe une présentation

$$
L^{\prime} \stackrel{u}{\rightarrow} L \stackrel{v}{\rightarrow} M \rightarrow 0
$$

où $L^{\prime}$ et $L$ sont des modules libres de type fini. On a alors un diagramme commutatif:

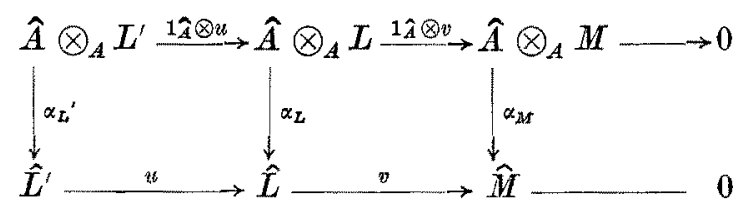

La première ligne est exacte car $\hat{A} \otimes_{A}$ : est exact à droite. D'après ce qui précède la seconde ligne est exacte et $\alpha_{L^{\prime}}, \alpha_{L}$ sont des isomorphismes, done $\alpha_{L}$ est bijectif d'après le lemme des cinq, ce qui démontre le point 4 ).

5) On termine maintenant la démonstration.

Pour tout idéal a de $A$ l'application canonique $\hat{A} \otimes_{A} \mathfrak{a} \rightarrow \hat{A}$ est injective comme composée de $\alpha_{\mathfrak{a}}$ et de l'injection $\hat{\mathfrak{a}} \rightarrow \hat{A}$, donc le $A$-module $a$ droite $\hat{A}$ est plat d'après un résultat de [3] (chap. $1, \S 2$, n. 3 , prop. 1 ). 
Supposons de plus que $\mathfrak{p}=\mathfrak{R}(A)$. Pour tout $a \in \mathfrak{R}(A) 1-a$ est inversible, d'après 2) pour tout module de type fini $M$ on $a \bigcap_{n \geqslant 0} R(A)^{n} M=0$ et $M$ est séparé pour la topologie $R(A)$-adique. Soit $M$ un $A$-module non nul, il existe $x \in M$ tel que $A x \neq 0$, le $A$-module $A x$ est séparé pour la topologie $\mathcal{R}(A)$-adique done $A x$ s'injecte dans $\hat{A} x$ et $\hat{A} x \simeq \hat{A} \otimes_{A} A x \neq 0$. Comme $\hat{A}$ est plat $\hat{A} \otimes_{A} M$ contient $\hat{A} \otimes_{A} A x$ et $\hat{A} \otimes_{A} M \neq 0$ pour tout $A$-module non nul $M$, i.e. $\hat{A}$ est fidélement plat.

2.4. Remarque. - Si $A$ est un anneau noethérien et si pour un idéal bilatère $\mathfrak{p}$ de $A$ la condition $R_{\mathfrak{p}}$ est vérifiée, on peut dèmontrer que pour entier $n$ on a $\hat{\mathfrak{p}}^{n}=\hat{\mathfrak{p}}^{n}$ dans le séparé compété $\hat{A}$ de $A$ pour la topologie $\mathfrak{p}$-adique.

2.5. Thtónkme. - Soit $B$ un $C 1$ anneau semi-local, soit $q$ un idéal bilatère de $B$ contenant $\mathfrak{R}(B)$, soit $A$ l'anneau $B / \bigcap_{n \geq 0} \mathfrak{q}^{n}$ et soit $\mathfrak{p}$ l'image de $\mathfrak{q}$ dans $A$. Soit Â le séparé compléte de $A$ (resp. B) pour la topologie $\mathfrak{p}$-adique (resp. $\mathfrak{q}$-adique), alors:

a) pour tout $A$-module de type fini $M$ le morphisme canonique $\hat{A} \otimes_{A} M \rightarrow \hat{M}$ où $\hat{M}$ est le complété séparé de M pour la topologie p-adique, est bijectif;

b) le A-module à droite $\hat{A}$ est plat;

c) si $\mathfrak{q}=\mathfrak{R}(B)$ alors tout A-module de type fini est séparé et $\hat{A}$ est fidèlement plat.

L'anneau $B$ est noethérien et tel que $B / \mathcal{R}(B)$ soit semi-simple donc pour tout entier $n$ l'anneau $B / R(B)^{n}$ est artinien et il en est même de $B / \mathfrak{q}^{n}$ donc aussi de $A / \mathfrak{p}^{n}$. Autrement dit la topologie $\mathfrak{p}$-adique du $O 1$ anneau noethérien $A$ est artinienne et séparée et d'après $2.2, A$ vérifie la condition $R_{\mathfrak{p}}$ et le théorème résulte du lemme 2.3.

\section{B) $Q$-anneaux noethériens quasi-locaux.}

\section{1. - Preliminaires.}

Dans toute cette partie, comme dans $A$, tous les anneaux sont noethériens.

On dit qu'un idéal premier $\mathfrak{p}$ d'un anneau $A$ est un idéal premier minimal si $\mathfrak{p}$ est un élément minimal de l'ensemble des idéaux premiers de $A$. On dit que $p$ est un idéal premier maximal si $\mathfrak{p}$ est un élément maximal de l'ensemble des idéaux premiers de $A$; comme un idéal bilatère maximal parmi l'ensemble des idéaux bilatères est premier, les idéaux premiers maximaux coïncident avec les idéaux bilatères maximaux.

Soit $M$ un $A$-module, on dit qu'un idéal premier $\mathfrak{p}$ est associé a $M$ si il existe un sous-module $N$ de $M$ tel que $p$ soit l'annulateur de tout sous-module non nul de $N$. Quand $A$ est un anneau noethérien pour tout module $M$ il existe un idéal bilatère, maximal parmi les annulateurs de sous-modules non nuls de $M$ et on vérifie facilement qu'un tel idéal est premier et associé à $M$. 
On désigne par $\operatorname{Ass}_{A}(M)$ on $\operatorname{Ass}(M)$ l'ensemble des idéaux premiers associés à $M$. Comme $A$ est noethérien $\operatorname{Ass}(M) \neq \emptyset$ pour $M \neq 0$. Il est facile de vérifier que si $M$ est coirréductible, alors Ass $(M)$ est réduit à un élément.

On aura souvent besoin des résultats suivants qui résultent des théorèmes 3.9 et 4.4 de [14] et du théorème 1 page 418 de [13].

\subsection{LEMME. - Soit $A$ un anneau noethérien semi-premier, alors:}

a) Un idéal $\mathfrak{a}$ de $A$ est essentiel dans A si et seulement si a contient un élément régulier;

b) L'anneau A a un anneau total des fractions à gauche, cet anneau est semisimple et coüncide aveo l'enveloppe injective de $A$.

En particulier, si $A$ est un anneau premier, tout idéal bilatère non nul est essentiel et contient done un élément régulier. De plus lorsque $A$ est premier, l'annean total des fractions à gauche de $A$ est simple.

On démontre maintenant une proposition relative à la structure de $E_{A}(A / \mathfrak{p})$ pour un idéal premier $\mathfrak{p}$ de $A$. L'assertion $a$ ) figure sans démonstration dans [29] page 61 .

1.2. Propositron. - Soit p un idéal premier de A, il existe un unique module injectif et indécomposable $F$ tel que:

a) $\operatorname{Ass}(F)=\{\mathfrak{p}\}$ et $E(A / \mathfrak{p})$ est isomorphe à $F^{n}$

b) si $Q=r_{F}(p)$ alors $Q$ n'a pas de sous-module quasi-injectif propre non nul.

1) Comme $A / p$ est monogène, $E(A / \mathfrak{p})$ est somme d'une famille finie $\left(F_{i}\right)_{1 \leqslant i \leqslant n}$ de modules injectifs indécomposables, i.e. $E(A / \mathfrak{p})=\oplus_{i=1}^{n} F_{i}$. Soit $i$ fixé, posons $\operatorname{Ass}\left(F_{i}\right)=$ $=\{\mathfrak{q}\}$, et soit $N \subset F_{i}$ tel que $\operatorname{Ann}\left(N^{\prime}\right)=\mathfrak{q}$ pour tout sous-module non nul $N^{\prime}$ de $N$. Par essentialité de $A / \mathfrak{p}$ dans $E(A / \mathfrak{p})$ on a $N \cap A / \mathfrak{p} \neq 0, \operatorname{donc} q=\operatorname{Ann}(N \cap A / \mathfrak{p})=\mathfrak{p}$; on a done pour tout $i \operatorname{Ass}\left(F_{i}\right)=\{\mathfrak{p}\}$.

2) Il résulte de II.1.2 que le module $B=r_{E(A / p)}(\mathfrak{p})=\bigoplus_{i=1}^{n} r_{F_{i}}(\mathfrak{p})$ est l'enveloppe injective du $A / \mathfrak{p}$-module $A / \mathfrak{p}$. Donc $B$ est l'anneau total des fractions à gauche de l'anneau premier noethérien $A / \mathfrak{p}$ et $B$ est un anneau simple d'après le théorème de Goldie. De plus pour tout $i$ le module $Q_{i}=r_{F_{i}}(\mathfrak{p})$ est non nul car $\{\mathfrak{p}\}=\operatorname{Ass}\left(F_{i}\right)$ et $Q_{i}$ est un $A / p$-module injectif.

3) Montrons que $Q_{i}$ est un $B$-module.

Le sous $A / p$-module singulier de l'anneau premier noethérien $A / p$ est nul, donc $B$ et les $Q_{i}$ sont des $A / p$-modules à sous-module singulier nul. Soit $c$ un élé. ment régulier de $A / p$, et soit $x \in Q_{i}$; le $A / p$-module $Q_{i}$ est injectif done le morphisme $a c \rightarrow a x$ de $(A / \mathfrak{p}) c$ dans $Q_{i}$ s'écrit $a c \rightarrow a c y$ pour $y \in Q_{i}$ et $x=c y$. De plus $y$ est uni- 
quement déterminé par cette condition ear si $c y=c y^{\prime}$ pour $y^{\prime} \in Q_{i}$ alors $c \in$ $\in \operatorname{Ann}\left(y-y^{\prime}\right)$, et $\operatorname{Ann}\left(y-y^{\prime}\right)$ est essentiel dans $A / \mathfrak{p}$, done $y=y^{\prime}$ car le sous-module singulier de $Q_{i}$ est nul. Comme tout élément de $B$ s'écrit $e^{-1} a$ avec $a \in A / p$ et $c$ régulier dans $A / \mathfrak{p}$ on vérifie aisément que $Q_{i}$ est un $B$-module en définissant le produit de $o^{-1} a \in B$ et de $x \in Q_{i}$ par $c^{-1} a x=z$ où $z$ est tel que $a x=c z$.

4) Comme $Q_{i}$ est un $A / p$-module coirréductible, $Q_{i}$ est à fortiori un $B$-module coirréductible, autrement dit $Q_{i}$ est un idéal coirréductible de l'anneau simple $B$, donc $Q_{i}$ est un $B$-module simple, et ceci prouve que les $Q_{i}$ sont des idéaux simples de $B$. Comme $B$ est un anneau simple, tous les $Q_{i}$ sont isomorphes comme $B$-module, done aussi comme $A$-module, donc tous les $F_{i}$ sont des $A$-modules isomorphes puisque $F_{i}=E_{A}\left(Q_{i}\right)$.

Ceci achève la démonstration de $a$ ).

5) Montrons maintenant $b$ ). Soit $i$ fixé, montrons que $Q_{i}$ ne contient aucun sous-module quasi-injectif propre non nul.

Soit $N_{i}$ un sous- $A$-module quasi-injectif non nul de $Q_{i}$, alors $N_{i}$ est un sous- $A / p-$ module quasi-injectif de $Q_{i}$. Pour $j \neq i Q_{j}$ est un $A / p$-module isomorphe à $Q_{i}$ et $Q_{j}$ contient un sous- $A / \mathfrak{p}$-module quasi-injectif $N_{\text {; }}$ isomorphe à $N_{i}$. Comme les $A / \mathfrak{p}-$ modules $Q_{j}$ sont coirrédueitibles, $N_{j}$ est essentiel dans $Q_{j}$ et le module $N=\oplus_{j=1}^{n} N_{j}$ est essentiel dans $B=\bigoplus_{j=1}^{n} Q_{j}$, comme $A / p$-module; donc $N \cap A / p$ est un idéal à gauche essentiel de l'anneau premier $A / \mathfrak{p}$ et il existe un élément régulier $e$ de $A / p$ tel que $c \in N$. De plus comme les modules quasi-injectifs $N_{j}$ sont tous isomorphes, $N$ est quasi-injectif.

On montre maintenant que $N=B$.

Soit $b \in B$, comme $N$ contient un élément régulier $e$, l'application $\lambda e \rightarrow \lambda b$ de $(A / \mathfrak{p}) c$ dans $B$ est un $A / \mathfrak{p}$-morphisme qui se prolonge en un endomorphisme $u$ du $A / \mathfrak{p}$ module injectif $B$; le sous-module quasi-injectif et essentiel $N$ de $B$ est invariant par $u$ et $b=u(c) \in N$. Ceci montre que $N=B$; on a done aussi $N_{i}=Q_{i}$ et ceci achève la démonstration de l'existence de $F$ vérifiant $a$ ) et $b$ ).

L'unicité de $F$ résulte de l'unicité de la décomposition d'un module injectif en modules injectifs indécomposables.

Soit $\gamma$ un ensemble de représentants des types de $A$-modules injectifs et indécomposables. Pour $F \in \mathcal{J}$ tel que $\{\mathfrak{p}\}=\operatorname{Ass}_{A}(F)$ on pose $\varphi_{A}(F)=$ p et $\varphi_{A}$ définit une application entre $\tilde{z}$ et l'ensemble des idéaux premiers de $A$. D'après 1.2 pour tout idéal premier $\mathfrak{p}$ on a $\operatorname{Ass}_{A}\left(E_{A}(A / \mathfrak{p})\right)=\{\mathfrak{p}\}$ et $\varphi_{A}$ est surjective. En général l'applieation $\varphi_{A}$ n'est pas injective, on a cependant le résultat classique suivant:

1.3. LeMne ([20] proposition 3.1). - Si A est un anneau noethérien commutatif alors l'application $\varphi_{A}$ est bijective.

On a également le résultat suivant qui nous servira au numéro 3 :

5- Annai di Matematica 
1.4. Propostrion. - Si la correspondance $\varphi_{A}$ est bijective pour l'anneau $A$ alors pour tout anneau quotient $A^{\prime}$ de $A$ la correspondance $\varphi_{A^{\prime}}$ est bijective.

Soit $A^{\prime}=A / \mathfrak{a}$ un quotient de $A$ par un idéal bilatère a. Soient $\mathfrak{p}^{\prime}=\mathfrak{p} / \mathfrak{a}$ un idéal premier de $A^{\prime}$ où p est un idéal premier de $A$, et $F, G$ deux $A^{\prime}$-modules injectifs indécomposables auxquels $\mathfrak{p}^{\prime}$ est associé. Il est immédiat de vérifier que $F$ et $G$ sont des $A$-modules quasi-injectifs et coirréductibles et que $\mathfrak{p}$ est associé aux $A$-modules $E_{A}(F)$ et $E_{A}(G)$. Comme $\varphi_{A}$ est bijective on a $E_{A}(F)=E_{A}(G)$. Considérons $F \oplus G$, comme $A^{\prime}$-module $F \oplus G$ est injectif, done quasi-injectif, et $F \oplus G$ est aussi quasi-injectif comme $A$-module. La proposition 2.4 de [15] montre que les $A$-modules $F$ et $G$ sont sont isomorphes, donc les $A^{\prime}$-modules $F$ et $G$ sont isomorphes et $\varphi_{A^{\prime}}$ est injective done bijective.

2. - La condition $C_{0}$.

2.1. LEMME. - Soit $F$ un module injectif indécomposable, posons $\Lambda s s(F)=\{\mathfrak{p}\}$, si $F$ n'a pas de sous-module quasi-injectif propre non $n u l$, alors $\mathfrak{p}=\mathfrak{l}_{A}\left(F^{\prime}\right)$ et $\mathfrak{p}$ est un idéal premier minimal.

Soit $N$ un sous-module non nul de $F$ tel que $\mathfrak{p}=\mathfrak{l}_{A}(N)$ il est immédiat de vérifier que $r_{F}(\mathfrak{p})$ est un sous-module caractéristique de $F$, done $r_{F}(p)$ est un sous-module quasi-injectif non nul de $F$ et on a $F=r_{F}(\mathfrak{p})$. Comme on a toujours $\mathfrak{l}_{A}\left(F^{\prime}\right) \subset \mathfrak{p}$ on a $\mathfrak{l}_{A}(F)=\mathfrak{p}$.

Montrons maintenant que $\mathfrak{p}$ est un idéal premier minimal. Soit $\mathfrak{q}$ un idéal premier tel que $\mathfrak{q} \subset \mathfrak{p}$. On a $\mathfrak{q} F=0$ et $F$ est donc un $A / \mathfrak{q}$-module injectif (II.1.2). Si $\mathfrak{q} \neq \mathfrak{p}$ l'idéal $\mathfrak{p} / \mathfrak{q}$ de l'anneau premier noethérien $A / \mathfrak{q}$ est bilatère done $\mathfrak{p} / \mathfrak{q}$ est un idéal essentiel dans $A / \mathfrak{q}$ et $\mathfrak{p} / \mathfrak{q}$ contient un élément régulier $c$ de l'anneau $A / \mathfrak{q}(1.1)$. Comme $F$ est un $A / \mathfrak{q}$-module injectif tout élément de $F$ est divisible par $e$, mais ceci est absurde car $c \in \mathfrak{p} / \mathfrak{q}$ et comme $\mathfrak{p} F=0$ on a $c F=0$. Cette absurdité montre que $\mathfrak{q}=\mathfrak{p}$ et $\mathfrak{p}$ est premier minimal.

2.2. DéfINITION. - On dit qu'un anneau $A$ vérifie la condition $C_{0}$ si pour tout idéal premier minimal et non maximal $\mathfrak{p}$ tout module injectif indécomposable $F$ tel que $\{p\}=$ $=\operatorname{Ass}_{A}(F)$ n'a pas de sous-module quasi-injectif propre non nul.

Cette définition est justifiée par la proposition suivante:

2.3. Propostrion. - Soit A un Q-anneau noethérien tel que $\bigcap_{n \geqslant 0} \mathcal{R}^{n}=0$, alors:

a) l'anneau $A$ vérifie la condition $C_{0}$;

b) tout idéal premier non maximal de $A$ est un idéal premier minimal.

2.3.1. - On démontre d'abord que si $\mathfrak{p}$ est un idéal premier de $A$ tel que $R \neq p$ alors $\mathfrak{p}$ est un idéal premier minimal et pour tout injectif indécomposable $F$ tel que $\{\mathfrak{p}\}=\operatorname{Ass}_{A}(F)$ le module $F$ n'a pas de sous-module quasi-injectif propre non nul. 
Soit $\mathfrak{p}$ un idéal premier tel que $R \not \mathfrak{p}$, et soit $F$ un module injectif indécomposable tel que $\{\mathfrak{p}\}=\operatorname{Ass}_{A}(F)$. Il existe un sous-module $N$ de $F$ tel que pour tout sous-module $N^{\prime} \neq 0$ de $N$ on ait $\mathfrak{I}_{A}\left(N^{\prime}\right)=\mathfrak{p}$. Soit $Q$ un sous-module quasi-injectif non nul de $F$ et considérons $M=E \oplus Q$ où $E$ est le cogénérateur minimal, comme $E$ est fidèle $M$ est fidèle. Montrons que $M$ est quasi-injectif.

D'après 1.2.1.1 il suffit de montrer que $M$ est stable par les endomorphismes de $E(M)=E \oplus F$ et pour cela il suffit de montrer que tout morphisme $f: E \rightarrow F$ est nul. Soit donc $f: E \rightarrow F$ et $x \in E$ tel que $f(x) \neq 0$, il existe $a \in A$ tel que $a f(x) \in$ $\in N-\{0\}$, et d'après II.1.5 il existe $n$ tel que $\mathfrak{R}^{n} a x=0$. On a done les relations suivantes:

$$
\mathfrak{R}^{n} \subset \mathfrak{l}_{A}(A a x) \subset \mathfrak{I}_{A}(A a f(x))=\mathfrak{p}
$$

Ceci prouve que $R \subset \mathfrak{p}$ et contredit l'hypothèse done $f=0$ et $M$ est quasi-injectif. Comme $A$ est un $Q$-anneau, le module quasi-injectif et fidèle $M$ est injectif donc $Q$ est injectif et $Q=F$. Autrement dit, $F$ n'a pas de sous-module quasi-injectif propre non nul, et d'après $2.1 \mathrm{p}$ est premier minimal.

2.3.2. - Montrons maintenant qu'un idéal premier non maximal de $A$ ne contient pas le radical de $A$. Compte tenu de 2.3 .1 ceci terminera la démonstration de la proposition.

L'anneau $A / \mathcal{R}$ est produit fini d'anneaux quasi-simples (II.3.3), par ailleurs un anneau quasi simple est premier et il est facile de vérifier que les idéaux premiers d'un produit fini d'anneaux quasi-simples sont exactement les idéaux bilatères maximaux. Autrement dit tout idéal premier de $A$ qui contient $R$ est un idéal bilatere maximal, i.e. un idéal premier maximal et ceci démontre 2.3.2.

On donne maintenant deux propositions de caractère technique qui seront utilisés dans le numéro suivant.

\subsection{Propositron. - Soit A un anneau noethérien,}

1) Si A vérifie la condition $\left(C_{0}\right)$ alors $A$ vérifie la condition suivante: $\left(C_{0}^{\prime}\right)$. pour tout idéal premier minimal et non maximal $\mathfrak{p}$ l'anneau total des fractions $d$ gauche de $A / \mathfrak{p}$ est $E_{A}(A / \mathfrak{p})$;

2) Si la correspondance $\varphi_{A}$ entre types d'injectifs indécomposables et idéaux premiers, est bijective alors les conditions $\left(C_{0}\right)$ et $\left(C_{0}^{\prime}\right)$ sont équivalentes.

1) Soit $\mathfrak{p}$ un idéal premier minimal et non maximal de $A$, d'après 1.2 il existe un module injectif indécomposable $F^{\prime}$ tel que $\operatorname{Ass}(F)=\{\mathfrak{p}\}$ et $E_{A}(A / \mathfrak{p})=F^{n}$. Comme $A$ vérifie $\left(C_{0}\right)$ on a $\mathfrak{p} F=0$ d'après 2.1 done $\mathfrak{p} E_{A}(A / \mathfrak{p})=0$ et d'après II.1.2 on a $E_{A / \mathfrak{p}}(A / \mathfrak{p})=E_{A}(A / \mathfrak{p})$ et la conclusion résulte de ce que $E_{A / \mathfrak{p}}(A / \mathfrak{p})$ est l'anneau total des fractions à gauche de $A / \mathfrak{p}$ (1.1).

2) On suppose que la correspondance $\varphi_{A}$ est bijective, il suffit de montrer que la condition $\left(C_{0}^{\prime}\right)$ implique la condition $\left(O_{0}\right)$. Soit $\mathfrak{p}$ un idéal premier minimal et non 
maximal de $A$ et soit $F$ un module injectif indécomposable anquel $\mathfrak{p}$ est associé, comme $\varphi_{A}$ est bijective il résulte de 1.2 que $E_{A}(A / p)$ est isomorphe à une somme finie de copies de $F$, si $\left(C_{0}^{\prime}\right)$ est vérifiée alors $E_{A}(A / \mathfrak{p})$ est un $A / \mathfrak{p}$-module done $\mathfrak{p} E_{A}(A / \mathfrak{p})=0$ et $\mathfrak{p} F=0$. On a alors $F=r_{F}(\mathfrak{p})$ et 1.2 montre également que $F$ n'a pas de sousmodule quasi-injectifi propre non nul, done $A$ vérifie la condition $\left(C_{0}\right)$.

2.5. Proposmion. - Soit A un anneau tel que tout idéal premier de A soit maximal ou minimal, si $A$ vérifie $\left(C_{0}\right)$ alors tout anneau quotient de $A$ vérifie $\left(C_{0}\right)$.

Soit a un idéal bilatère de $A$ et soit $A^{\prime}=A / \mathfrak{a}$ un quotient de $A$, soit $\mathfrak{p}^{\prime}$ un idéal premier minimal et non maximal de $A^{\prime}$, alors $\mathfrak{p}^{\prime}=\mathfrak{p} / \mathfrak{a}$ où $\mathfrak{p}$ est un idéal premier de $A$, non maximal et par suite minimal. Soit $F^{\prime}$ un $A^{\prime}$-module injectif indécomposable tel que $\left\{\mathfrak{p}^{\prime}\right\}=\mathrm{Ass}_{A^{\prime}}\left(F^{\prime}\right)$, soit $F=E_{A}\left(F^{\prime}\right)$; on vérifie immédiatement que $F$ est un $A$-module injectif indécomposable tel que $\{\mathfrak{p}\}=\operatorname{Ass}_{A}(F)$, done avec 2.1, $F$ n'a pas de sous-module quasi-injeetif propre non nul. Or $F^{\prime}$ est un sous-module quasi-injectif non nul de $F$, on a done $F^{\prime}=F$ et $A^{\prime}$ vérifie la condition $\left(C_{0}\right)$.

Les résultats précédents vont maintenant être utilisés pour caractériser certains $Q$-anneaux quasi-loeaux.

3. - Caracterisation des Q-anneaux locaux.

Soit $A$ un $Q$-anneau noethérien de radical $\mathcal{R}$ tel que $\bigcap_{n \geqslant 0} \mathfrak{R}^{n}=0$ alors $A$ est un $C 1$ anneau et $A$ vérifie la condition $C_{0}$ et la condition $b$ ) de 2.3. On va démontrer ici une réciproque de ce résultat lorsque $A$ est un anneau local tel que la correspondance $\varphi_{A}$ soit bijective.

Remarquons d'abord que lorsque $A$ est local, le radical $\mathcal{R}$ de $A$ est l'unique idéal bilatère maximal de $A$ et $\mathcal{R}$ est un idéal premier. De plus comme tout idéal bilatère est contenu dans un idéal bilatère maximal, $R$ contient tous les idéaux bilatères de $A$ et en particulier $\mathfrak{R}$ contient tous les idéaux premiers.

3.1. THÉORÈye. - Soit $A$ un anneau local tel que la correspondance $\varphi_{A}$ entre types d'injectifs indécomposables et idéaus bilatères premiers soit bijective, alors $A$ est un Q-anneau si et seulement si A vérifie les conditions suivantes:

a) A est un 01 anneau;

b) pour tout idéal premier $\mathfrak{p}$ distinct de $\mathfrak{R}$ lanneau total des fractions à gauche de l'anneau $A / \mathfrak{p}$ coincide avee $B_{A}(A / \mathfrak{p})$.

Les conditions sont nécessaires: Si $A$ est un $Q$-anneau alors $A$ est un $C 1$ anneau et $A$ vérifie la condition $b$ ) d'après 2.3 et 2.4 .

Les conditions sont suffisantes: Nous commençons par démontrer le résultat suivant:

3.1.1. - Si $A$ est un anneau local vérifiant les conditions $a$ ) et $b$ ) ci-dessus et si la correspondance $\varphi_{A}$ est bijective alors tout $A$-module quasi-injectif fidèle est injectif. 
Soit $\mathfrak{p}$ un idéal premier, il existe un unique module injectif indécomposable auquel $\mathfrak{p}$ est associé. Si $\mathfrak{p} \neq \mathcal{R}$ notons $F_{\mathfrak{p}}$ l'injectif indécomposable tel que $\{\mathfrak{p}\}=\operatorname{Ass}\left(F_{\mathfrak{p}}\right)$; d'après 1.2 on peut écrire $E_{A}(A / \mathfrak{p}) \simeq F_{\mathfrak{p}}^{n}$ pour un entier $n$, d'après $b$ ) on a $\mathfrak{p} E_{A}(A / \mathfrak{p})=$ $=0$ donc $\mathfrak{p} F_{\mathfrak{p}}=0$ et d'après $1.2 F_{\mathfrak{p}}=r_{F_{\mathfrak{p}}}(\mathfrak{p})$ n'a pas de sous-module quasi-injectif propre non nul.

Etudions le cas $\mathfrak{p}=\mathcal{R}$. Comme $A$ est local, il $y$ a un seul type de $A$-module simple, soit $S$ un $A$-module simple, alors $E=E(S)$ est le cogénérateur minimal et $E$ est l'unique injectif indécomposable auquel $\mathcal{R}$ est associé.

Soit $N$ un module quasi-injectif, décomposons $N$ en somme directe de modules quasi-injectifs et indécomposables. Deux facteurs de cette somme qui ont la même enveloppe injective sont isomorphes d'après la proposition 2.4 de [15] et on peut écrire

$$
N=N_{0}^{(\mathcal{y})} \oplus \underset{\mathfrak{p} \subseteq \Omega}{\oplus} F_{\mathfrak{p}}^{\left(\tilde{f}_{p}\right)}
$$

où $\Omega$ est contenu dans l'ensemble des idéaux premiers distincts de $R$, et où $N_{0}$ est un sous-module quasi-injectif de $E ; \Omega$ peut être vide et $N_{0}$ peut être réduit à 0 .

Supposons que $N$ ne soit pas injectif et montrons que $N$ n'est pas fidèle ce qui démontrera 3.1.1.

Si $N$ n'est pas injectif alors $N_{0} \neq 0$ et $N_{0}$ n'est pas injectif done $N_{0} \neq E$. Distinguons deux cas suivant que $\Omega=\emptyset$ ou $\Omega \neq \emptyset$.

(i) Si $\Omega=\emptyset$ alors $N=N_{0}^{(\mathfrak{\gamma})}$, comme $N_{0}=r_{E}\left(\mathfrak{l}_{A A}\left(N_{0}\right)\right)$ d'après la condition $C 1$ et comme $N_{0} \neq E$ on a $\mathfrak{l}_{A}\left(N_{0}\right) \neq 0$ done $N$ n'est pas fidèle car $\mathfrak{I}_{A}(N)=\mathfrak{l}_{A}\left(N_{0}\right)$.

(ii) Si $\Omega \neq \emptyset$ montrons d'abord que pour $\mathfrak{p} \in \Omega$ on $a r_{E}(\mathfrak{p}) \subset N_{0}$. Le module $N_{0} \oplus F_{\mathfrak{p}}$ est quasi-injectif comme facteur direct de $N$ done pour $n$ entier $N_{0}^{n} \oplus F_{\mathfrak{p}}^{n}$ est quasi-injectif et $N_{0} \oplus F_{\mathfrak{p}}^{n}$ est aussi quasi-injectif. Si on prend $n$ tel que $F_{\mathfrak{p}}^{n} \simeq$ $\simeq E_{A}(A / \mathfrak{p})$ on voit que $N_{0} \oplus E_{A}(A / \mathfrak{p})$ est quasi-injectif. Le module $N_{0} \oplus E_{A}(A / \mathfrak{p})=$ $=M$ est done stable par les endomorphismes de son enveloppe injective $E \oplus E_{A}(A / \mathfrak{p})$, soit $x \in E$ tel que $\mathfrak{p} x=0$, on a un morphisme non nul $f: A / \mathfrak{p} \rightarrow A / \operatorname{Ann}(x) \rightarrow E$ tel que $x=f(1+\mathfrak{p})$, et $f$ se prolonge en un morphisme non nul $u: E_{A}(A / \mathfrak{p}) \rightarrow E$ tel que $x \in u\left(E_{A}(A / \mathfrak{p})\right)$, si $v$ est l'endomorphisme de $E \oplus E_{A}(A / \mathfrak{p})$ obtenu en prolongeant $u$ par 0 sur $E$ on a $v(M) \subset M$ done $u\left(E_{A}(A / \mathfrak{p})\right) \subset N_{0}$ et $x \in N_{0}$. Ceci montre que $r_{E}(\mathfrak{p}) \subset N_{0}$ et d'après I.1.1. et 2.1 on a

$$
\mathfrak{l}_{A}\left(N_{0}\right) \subset \mathfrak{l}_{A}\left(r_{E}(\mathfrak{p})\right)=\mathfrak{p}=\mathfrak{l}_{A}\left(F_{\mathfrak{p}}\right)
$$

Il résulte de ces relations que $\mathfrak{I}_{A}\left(N_{0}\right)=\mathfrak{I}_{A}(N)$, comme $N_{0} \neq E$ on a $\mathfrak{I}_{A}\left(N_{0}\right) \neq 0$ de la même façon qu'en (i) et ceci montre que $N$ n'est pas fidèle et achève la démonstration de 3.1.1.

3.1.2. - On montre maintenant que $A$ est un $Q$-anneau en montrant que pour tout idéal bilatère $\mathfrak{a}$ de $A$ tout $A / \mathfrak{a}$-module quasi-injectif et fidèle est injectif (I.2.3.4). 
Soit $A^{\prime}=A / \mathfrak{a}$ un anneau quotient de $A . A^{\prime}$ est évidemment un anneau local, la correspondance $\varphi_{A^{\prime}}$ est bijective d'après 1.4, et $A^{\prime}$ est un $O 1$ anneau d'après II.2.1 et pour montrer que tout $A^{\prime}$-module quasi-injectif et fidèle est injectif il suffit d'après 3.1.1 de montrer que $A^{\prime}$ vérifie l'analogue de la condition $b$ ) de 3.1. On a vu dans la démonstration de 3.1.1 que pour tout idéal premier $\mathfrak{p} \neq \Re$ l'injectif indécomposable $F_{\mathfrak{y}}$ tel que $\{\mathfrak{p}\}=\operatorname{Ass}\left(F_{\mathfrak{p}}\right)$ n'a pas de sous-module quasi-injectif propre non nul et d'après 2.1 ceci montre que tout idéal premier $\mathfrak{p} \neq \mathfrak{R}$ est premier minimal, autrement dit la condition $b$ ) de 3.1 n'est pas autre chose que la condition $\left(O_{0}^{\prime}\right)$ de la proposition 2.4. La même proposition 2.4 montre que $A$ vérifie $\left(O_{0}\right)$ et d'après 2.5 l'anneau $A^{\prime}$ vérifie aussi $\left(C_{0}\right)$ une derniére utilisation de 2.4 montre que $A^{\prime}$ vérifie la condition $b$ ) de la proposition 3.1 et ceci achève la démonstration.

\subsection{ReMARque.}

1) Il est facile de généraliser la proposition 3.1 à un anneau quasilocal, mais cette généralisation n'est qu'apparente car on peut démontrer aisément qu'un anneau quasi-local $A$ tel que la correspondance $\varphi_{A}$ soit bijective est en fait local.

2) Il existe des $Q$-anneanx $A$ tels que la correspondance $\varphi_{A}$ ne soit pas bijective' par exemple l'anneau $A$ défini en II.3.4 est un $Q$-anneau dans lequel $\mathcal{O}$ est le seul idéal premier, mais il $y$ a deux types de modules injectifs indécomposables: l'enveloppe injective de $A$ et l'enveloppe injective de l'unique type de $A$-module simple (cf. [31] exposé 20).

Voici un exemple de $Q$-anneau local pas nécessairement commutatif tel que $\varphi_{A}$ soit bijective.

3.3. Proposttion. - Soit A un anneau local sans diviseur de 0 dont le radical $\mathfrak{m}$ est un idéal à gauche principal et tel que $A / \mathrm{m}$ soit un corps, alors $A$ est un $Q$-anneau.

Montrons que $A$ vérifie les conditions du théorème 3.1. Posons $\mathrm{m}=A a$.

1) D'après la proposition 2.1 de [25] tout idéal à gauche de $A$ est bilatère et de la forme $\mathfrak{m}^{n}=A a^{n}$. Il résulte alors du théorème 3.5 de [18] que la correspondance $\varphi_{A}$ est bijective.

2) L'idéal $\mathcal{O}$ est le seul idéal premier de $A$ autre que m et il est clair que $A$ vérifie la condition $b$ ) de 3.1 .

3) Montrons que $A$ vérifie la condition $C 1$.

Soit $E=E_{A}(A / \mathfrak{m})$ le cogénérateur minimal de $\operatorname{Mod} A$. Montrons d'abord que $E=\bigcup_{n \geqslant 1} r_{E}\left(A a^{n}\right)$ en montrant que $M=\bigcup_{n \geqslant 1} r_{E}\left(A a^{n}\right)$ est injectif. Soit $f$ un morphisme d'un idéal à gauche $A a^{n}$ de $A$ dans $M$ et soit $x=f\left(a^{n}\right)$, le module injectif $E$ est divisible et comme $a^{n}$ est régulier il existe $y \in E$ tel que $x=a^{n} y$. Comme $x \in M$ il existe $m$ tel que $a^{m} x=0$ et on a $a^{n+m} y=0$, done $y \in M$ et $M$ est injectif. Comme $M$ est essentiel dans $E$ on a $M=E$. 
Montrons maintenant que les modules $r_{E}\left(A a^{n}\right)$ sont les seuls sous-modules quasiinjectifs de $E$.

D'abord pour tout $x \in E$ il existe un unique $n$ tel que $\operatorname{Ann}(x)=A a^{n}$; en effet il existe un unique $n$ tel que $x \in r_{E}\left(A a^{n}\right)-r_{E}\left(A a^{n-1}\right)$ et on a $A a^{n} \subset \operatorname{Ann}(x){ }_{\neq}^{C} A a^{n-1}$ done $\operatorname{Ann}(x)=A a^{n}$. Soit maintenant $N$ un sous-module quasi-injectif de $E$, supposons $N \neq E$, alors il existe $n$ tel que $r_{E}\left(A a^{n}\right) \notin N$. Soit $m$ le plus grand entier tel que $r_{E}\left(A a^{m}\right) \subset N$, si $r_{E}\left(A a^{m}\right) \neq N$ il existe $x \in N$ tel que $a^{m} x \neq 0$ et il existe $p \geqslant 1$ tel que $\operatorname{Ann}(x)=A a^{m+p}$. Soit alors $y \in r_{E}\left(A a^{m+1}\right)$ on a $\operatorname{Ann}(x) \subset \operatorname{Ann}(y)$ et il existe un morphisme $A x \rightarrow A y$ qui se prolonge en un endomorphisme $u$ de $E$, comme $N$ est quasi-injectif on a $u(N) \subset N$ et $y=u(x) \in N$. On a done $r_{E}\left(A a^{m+1}\right) \subset N$, ce qui contredit la définition de $m$ et par conséquent $N=r_{E}\left(A a^{m}\right)$. Comme pour tout entier $n$ on a $A a^{n}=\mathfrak{l}_{A} r_{E}\left(A a^{n}\right)$ il est clair que $A$ vérifie la condition $C 1$.

\section{4. - D'autres exemples de Q-anneau.}

Nous commençons par donner des conditions suffisantes pour qu'un $C 1$ anneau noethérien soit quasi-local.

4.1. LEMrne. - Soit A un C1 anneau noethérien, A est quasi-local dès que l'une des conditions suivantes est vérifiée:

1) pour tout idéal bilatère maximal $\mathfrak{p}$ on $a \bigcap_{n \geqslant 0} \mathfrak{p}^{n}=0$;

2) A est premier et il existe un idéal bilatère maximal $\mathfrak{p}$ tel que $\bigcap_{n \geqslant 0} \mathfrak{p}^{n}=0$.

Si $A$ est quasi-simple, il n'y a rien à rien à démontrer, on peut done supposer que $A$ n'est pas quasi-simple et $A$ possède alors des idéaux bilatères maximaux.

a) Montrons d'abord que $A$ a un unique idéal bilatère maximal.

Soit $\mathfrak{p}$ un idéal bilatère maximal tel que $\bigcap_{n \geqslant 1} \mathfrak{p}^{n}=0$. Soit $E$ le cogénérateur minimal de $\operatorname{Mod} A$. Le module $E_{(\mathfrak{p})}=\bigcup_{n \geqslant 1} r_{E}\left(\mathfrak{p}^{n}\right)$ est un sous-module quasi-injectif et fídèle de $E \operatorname{car} \mathfrak{I}_{A}\left(E_{(\mathfrak{p})}\right)=\bigcap_{n \geqslant 1} \mathfrak{l}_{A} r_{E}\left(\mathfrak{p}^{n}\right)=\bigcap_{n \geqslant 1} \mathfrak{p}^{n}=0$ (II.1.1), done $E_{(\mathfrak{p})}$ est injectif car $A$ est un $C 1$ anneau. De plus en notant $\mathcal{S}_{(\mathfrak{p})}$ le sous-module du socle de $E$ annulé par $\mathfrak{p}$ on a $E_{(\mathfrak{p})}=E_{A}\left(\S_{\mathfrak{p})}\right)$ (II.1.3 et II.1.4). Soit $\mathfrak{q}$ un idèal bilatère maximal distinct de $\mathfrak{p}$, on va montrer que cette hypothèse est absurde ce qui démontrera $a$ ).

Soit $S$ un sous-module simple de $E$ annulé par $\mathfrak{q}$, on a $\mathfrak{q}=\mathfrak{l}_{A}(S)$, montrons que le module $N=E_{(\mathfrak{p})} \oplus S$ est injectif. Tout sous-module simple de $S_{(\mathfrak{p})}$ est annulé par $\mathfrak{p}$ et comme $\mathfrak{I}_{A}(S)=\mathfrak{q}$ le module $S$ n'est isomorphe à aucun sous-module de $\boldsymbol{S}_{(\mathfrak{p})}$ et ainsi $N$ est un sous-module de $E$; le module $N$ est fidèle car $E_{(\mathfrak{p})}$ est fidèle, et, pour montrer que $N$ est injectif, il suffit de montrer que $N$ est quasi-injectif car $A$ est un $C 1$ anneau. Montrons que $N$ est quasi-injectif. Soit $T_{\mathfrak{p}}$ l'ensemble des sousmodules simples de $\delta_{(\mathfrak{p})}$, on a $\delta_{(\mathfrak{p})}=\underset{U \in T_{\mathfrak{p}}}{\oplus} U(\mathbf{I I} .1 .3)$ et $E_{(\mathfrak{p})}=\underset{U \in T_{\mathfrak{p}}}{\oplus} E(U)$ car $A$ est noethérien. Soit $U \in T_{\mathfrak{p}}$, soit $f: E(U) \rightarrow E(S)$ un morphisme non nul, il existe $x \in E(U)$ 
tel que $f(x) \neq 0$, il existe a $\in A$ tel que $a f(x) \in S-\{0\}$ et il existe $n$ tel que $\mathfrak{p}^{n} \subset$ $\subset \mathfrak{I}_{A}(A a x) \subset \mathfrak{I}_{A}(A a f(x))=\mathfrak{I}_{A}(S)=q$ et ceci est absurde car $\mathfrak{p}$ et $q$ sont des idéaux bilatères maximaux distincts. Autrement dit, tout morphisme de $E_{(\mathfrak{p})}$ dans $E(S)$ est nul et on vérifie alors facilement que $N$ est stable par tous les endomorphismes de son enveloppe injective $E(N)=E_{(p)} \oplus E(S)$ et ainsi $N$ est quasi-injectif.

Il résulte de ceei que $N$ est injectif done $S$ aussi, et ceci montre que tout sousmodule simple de $E$ annulé par q est injectif.

Pour terminer la démonstration de $a$ ) on distingue maintenant deux cas suivant que $\bigcap_{n \geqslant 1} \mathfrak{q}^{n}=0$ (condition 1)) ou que $A$ est premier (condition 2)).

1) Si $\bigcap_{n \geqslant 1} \mathfrak{q}^{n}=0$ alors en posant $E_{(\mathfrak{q})}=\bigcup_{n \geqslant 1} r_{E}\left(\mathfrak{q}^{n}\right)$ on a d'après II. 1.1 les relations: $\mathfrak{l}_{A}\left(E_{(\mathfrak{q})}\right)=\bigcap_{n \geqslant 1} \mathfrak{l}_{A}\left(r_{E}\left(\mathfrak{q}^{n}\right)\right)=\bigcap_{n \geqslant 1} \mathfrak{q}^{n}=0$. Il résulte de ce qui précède que le module $S_{(\mathfrak{q})}$ somme des sous-modules simples de $E$ annulés par q est injectif et d'après II.1.4 on a $\delta_{(\mathfrak{q})}=E_{(\mathfrak{q})}$, ce qui est absurde car $\mathfrak{q} \subset \mathfrak{I}_{A}\left(\delta_{(\mathfrak{q})}\right)=0$ et $\mathfrak{q}=0$, et ceci montre que la condition $a$ ) est vérifiée dans ce cass.

2) Si $E$ est premier, l'idéal bilatère q contient un élément régulier $e$ qui divise chaque module simple injectif $S$ annulé par $q$ ce qui est absurde et ceci démontre la condition $a$ ) dans ce cas et la démonstration de $a$ ) est terminée.

b) Montrons que $R$ est l'unique idéal bilatère maximal de $A$ ce qui prouvera que $A$ est quasi-local.

Comme tout idéal bilatère maximal contient le radical $\Re$ de $A$, il résulte de a) que l'anneau $A / \Omega$ a un unique idéal bilatère maximal. D'après I.2.4 et II.3.6, l'anneau $A / R$ est produit fini d'anneaux quasi-simples. Il est facile de vérifier qu'un produit de $n$ anneaux quasi- simples possède $n$ idéaux bilatères maximaux, et par conséquent $A / R$ est réduit à un seul anneau quasi-simple, i.e. $A / R$ est quasi-simple et la proposition est démontrée.

4.2. COROLLAIRE. - Soit A un C1 amneau noethérien commutatif, alors chaque idéal premier de A est contenu dans un unique idéal bilatère maximal.

Soit $\mathfrak{p}$ un idéal premier de $A$. Si $\mathfrak{p}$ est maximal, il n'y a rien à dèmontrer. Sinon l'anneau $A / p$ est un $C 1$ anneau d'après II.2.4 (i), noethérien intègre done pour tout idéal maximal $\mathrm{m}$ on $\mathrm{a} \bigcap_{n \geqslant 0} \mathrm{~m}^{n}=0$ et $A / p$ est un anneau local d'après 4.1 d'où le
corollaire.

Rappelons qu'un anneau $A$ est dit borné à gauche si tout idéal à gauche essentiel contient un idéal bilatère non nul. On a une notion analogue d'anneau borné à droite. Rappelons également qu'un anneau de valuation discrète est un anneau local $A$ sans diviseurs de 0 dont tous les idéaux à gauche et à droite sont principanx et tel que $A / \mathcal{R}(A)$ soit un corps, il existe alors $a \in A$ tel que $R(A)=A a=a A$ et tout idéal, à gauche ou à droite, de $A$ est de la forme $A a^{n}=a^{n} A$ ([25] proposition 2.1 et théorème 3.6 ). 
4.3. Proposition. - Soit $A$ un anneau local sans diviseurs de 0 dont tous les idéaux $\dot{a}$ gauche et tons les idéaux d̀ droite sont principaux, ators $A$ est un $Q$-anneau.

Si le radical $R$ de $A$ est nul alors $A$ est un corps et un $Q$-anneau. Supposons $R \neq 0$ et montrons que $A$ vérifie les conditions du théorème 3.1 .

D'abord $A$ est un anneau premier, noethérien et héréditaire des deux côtés dont le radical n'est pas nul dono $A$ est borné à gauche et à droite d'après le théorème 4.13 de [8] et il est facile de vérifier en utilisant le théorème 3.5 de [18] que la correspondance $\varphi_{A}$ est bijective. De plus l'idéal $\mathcal{O}$ est le seul idéal premier de $A$ autre que $R$ et il est clair que $A$ vérifie la condition $b$ ) de 3.1 .

Montrons que $A$ est un $C 1$ anneau.

D'après la proposition 2.3 et le lemme 2.10 de [21] l'anneau $A$ coïncide avec son enveloppe locale $L(R)$ qui est le sous-anneau du corps des fractions $Q$ de $A$ engendré par $A$ et les inverses dans $Q$ des éléments de $\mathrm{C}(\Re)=\{0 \in A \mid c x \in R \Rightarrow x \in \Re\}$. Soit $\hat{A}$ le séparé complété de $A$ pour la topologie $R$-adique, d'après les lemmes 2.1 et 2.2 de [19] l'anneau $\hat{A}$ est un anneau semi-local premier dont tous les idéaux à gauche et tous les idéaux à droite sont principaux et $\hat{A}$ est un anneau de matrices sur un anneau de valuation discrète $D$. Il résulte donc de 3.3 et de II.2.5 que $\hat{A}$ est un $Q$ anneau. De plus les lemmes 2.3 et 3.15 de [15] montrent qu'il n'y a qu'une classe de $\hat{A}$-modules simples et $\hat{A}$ est un anneau local. Ces lemmes montrent aussi que si $S$ est un $A$-module simple alors $S$ est un $\hat{A}$-module simple et le théorème 3.17 de [19] montre qu'alors $E_{A}(S)=E_{\hat{A}}(S)$.

On peut maintenant montrer que $A$ vérifie la condition $\mathrm{C} 1$.

Soit $N$ un sous-module quasi-injectif non nul de $E=E_{A}(S)=E_{\hat{A}}(S)$, alors $N$ est un sous-module de $E$ par définition de la structure de $\hat{A}$-module de $E$ ([19] lemme 3.14) et on vérifie aisément que $N$ est un sous- $\hat{A}$-module quasi-injectif de $E$, on a done $N=r_{E}\left(\Upsilon_{\hat{A}}(N)\right)$ car $\hat{A}$ est un $Q$-anneau. Comme $N$ est non nul $\Upsilon_{\hat{A}}(N)$ est un idéal bilatère de $\hat{A}$ et d'après le théorème 3.5 de $[25]$ il existe $n$ tel que $\mathfrak{I}_{\hat{A}}(N)=$ $=\Re(\hat{A})^{n}$.

D'après le lemme 1 de $[16]$ on a $R(\hat{A})^{n} \cap A=\Re^{n}$ et $R(\hat{A})^{n}=\hat{A} \Re^{n}$ et on peut écrire les égalités suivantes:

$$
N=r_{E}\left(\Re(\hat{A})^{n}\right)=r_{E}\left(R^{n}\right)=r_{E}\left(l_{\hat{A}}(N) \cap A\right)=r_{E}\left(\mathfrak{l}_{A}(N)\right)
$$

Comme $E$ est le cogénérateur minimal de Mod $A$ ceci montre que $A$ vérifie la condition 01 et la proposition 4.3 est démontrée.

Rappelons qu'un anneau de Dedekind premier est un anneau premier, noethérien et héréditaire des deux côtés, qui ne possède pas d'idéal bilatère propre idempotent [8].

4.4. Conollaire. - Soit $A$ un anneau de Dedekind premier borné (d̀ gauche et $\grave{a}$ droite) alors les assertions suivantes sont équivalentes:

a) $A$ est un Q-anneau, 
b) A est un $C 1$ anneau,

c) A est un anneau local.

Soit $A$ un anneau de Dedekind premier borné. On suppose que $A$ n'est pas un anneau simple sinon il n'y a rien à démontrer.

L'implication $a) \Rightarrow b$ ) est triviale, montrons que $b) \Rightarrow c$ ) et que $c \Rightarrow a) . b$ ) $\Rightarrow c$ ): Pour tout idéal bilatère maximal $\mathfrak{p}$ de $A$ on a $\bigcap_{n \geq 0} \mathfrak{p}^{n}=0$ d'après ([8] proposition 2.2 et lemme 4.1), donc d'après 4.1 l'anneau $A$ est quasi-local. Comme $A$ est borné et n'est pas un anneau simple, on a $\Re(A) \neq 0$ et il résulte de ([21] lemme 2.10 et propositions 2.3 et 2.6$)$ que $A / \mathcal{R}(A)$ est un anneau simple done $A$ est un anneau local.

c) $\Rightarrow a)$. Si $A$ est un anneau de Dedekind premier borné et local alors $A$ est un anneau de matrices sur un anneau $D$ sans diviseurs de 0 , a idéaux à gauche et à droite principanx, semi local et borné ([21] corollaire 3.10). En fait il est facile de vérifier que comme $A / \mathcal{R}(A)$ est simple $D / \mathcal{R}(D)$ est aussi simple car sinon un idempotent non nul et différent de 1 de $D / \mathscr{R}(D)$ permet de construire un idempotent central non nul et différent de 1 de $A / R(A)$ ce qui est absurde; ainsi $D$ est un anneau local. Il résulte alors de 4.3 que $D$ est un $Q$-anneau et $A$ est aussi un $Q$ anneau d'après II,2.5.

Dans un article ultérieur, nous étudierons plus précisément la structure des $C 1$ anneaux et des $Q$-anneaux noethériens commutatifs, dans ce cas le corollaire 4.2 permet de démontrer qu'un $C 1$ anneau noethérien est produit fini de $C 1$ anneaux locaux.

\section{BIBLIOGRAPHIE}

[1] H. BAss, Finitistic dimension and a homological generalisation of semi primary rings, Trans. Amer. Math. Soe., 95 (1960), pp. 466-488.

[2] N. BourBaKI, Algèbre, Chapitre 8, Hermann (1958).

[3] N. BovrBaki, Algèbre commutative, Chapitres 1, 2, 3, 4, Hermann (1961).

[4] N. BourbakI, Topologie générale, Chapitres 3 et 4, Hexmann (1960).

[5] K. A. BYRD, Rings whose quasi injective modules are injective, Proc. Amer. Math. Soc,, 33, 2 (1972), pp. $235 \cdot 240$.

[6] S. V. CHAse, Direct products of modules, Trans. Amer. Math. Soc., 97 (1960), pp. 457-473.

[7] J. H. Cozzess, Homological properties of the rings of differential polynomials, Bull. Amer. Math. Soc., 76, 1 (1970), pp. 75-79.

[8] D. Ersen Bud - J. C. Robson, Hereditary noetherian prime rings, J. of Algebra, 16 (1970), pp. $86-104$.

[9] C. FAITH, Rings with ascending condition on annihilators, Nagoya Math. J., 27 (1966), pp. $179-191$.

[10] C. FAITH, Lectures on injective modules and quotient rings, Springer-Verlag (1967).

[11] L. Fuchs, Annali della Scuola Norm. Sup. Pisa, Série III, 23 (1969), pp. 541. 
[12] K. R. Fuller, On direct representations of quasi-injectives and quasi-projectives, Arch. Math., 20 (1969), pp. 495-502.

[13] P. Gabriet, Des catégories abéliennes, Bull. Soc. Math. France, 90 (1962).

[14] A. W. Goldie, Semi prime rings with maximum condition, London Math. Soc., 10 (1960), pp. $589-608$.

[15] M. Harada, Note on quasi injective modules, Osaka J. Math., 2 (1965), pp. 351.356.

[16] Y. Hivoara, Note on noncommutative semilocal rings, Nogaya Math. J., 17 (1960), pp. 161.167.

[17] R. E. JoHnson - E. T. WoNG, Quasi injective modules and irreducible rings, J. London Math. Soc., 36 (1961), pp. 260-268.

[18] G. KRAUSE, On fully left bounded left noetherian rings, J. of Algebra, 23 (1972), pp. 88-99.

[19] H. Marubayashu, Modules over bounded Dedekind prime rings, Osaka J. Math., 9 (1972), pp. $95-110$.

[20] E. Matlis, Injective modules over noetherian rings, Pac. J. Math., 8 (1958), pp. 511.528.

[21] G. O. Mrchler, Asano Orders, Proc. London Math. Soc., (3), 19 (1969), pp. 421-443.

[22] A. PAGE, Théorie des anneaux associés, thèse, Fac. Sc. Poitiers (1972).

[23] N. Popescu - C. Nastasescu, Anneaux semi-artiniens, Bull. Soc. Math. France, 96 (1968), pp. 357-368.

[24] N. Popescu - T. Sprrov, Sur les épimorphismes plats d'anneaux, Comptes Rendus Ac. Sci. Paris, 268 (1969), pp. 376.

[25] G. A. Probert, Local rings whose maximal ideal is principal as a right ideal, Proc. London, Math. Soc., (3), 19 (1969), pp. 403-420.

[26] G. Renault, Anneaux réduits non commutatifs, J. Math. Pures et Appliquées, 46 (1967), pp. $203-214$

[27] G. RENAULT, Sur les anneaux tels que tout produit de copies d'un module quasi injectif soit quasi injectif, Comptes Rendus Ac. Sci., 271 (1970), pp. 12-15.

[28] G. Renault - A. Camlead, Exude des modules 2 -quasi-injectifs, Comptes Rendus Ac. Sc. Paris, 270 (1970), pp. 1391-1393.

[29] G. Renault - J. Fort, Séminaire d'algèbre, Fac. Sc. de Poitiers, (1967-1968).

[30] B. Roux, Un critère de décomposition d'un anneau en produit d'anneaux, Comptes Rendus Ac. Sc. Paris, 274 (1972), pp. 235-238.

[31] Séminaire d'Algèbre non commutative, Orsay, 1969-1970.

[32] C. Tisseron, Quelques propriétés des modules quasi-injectifs, Comptes Rendus Ac. Sc. Paris, 268 (1969), pp. 1377-1380. 